\title{
Molecules and dust production in the Magellanic Clouds ${ }^{\star}$
}

\author{
J. Th. van Loon $^{1}$, M. Cohen ${ }^{2}$, J. M. Oliveira ${ }^{1}$, M. Matsuura ${ }^{3,4}$, I. McDonald ${ }^{1}$, G. C. Sloan ${ }^{5}$, \\ P. R. Wood ${ }^{6}$, and A. A. Zijlstra ${ }^{7}$
}

1 Astrophysics Group, Lennard-Jones Laboratories, Keele University, Staffordshire ST5 5BG, UK e-mail: jacco@astro.keele.ac.uk

2 Radio Astronomy Lab, 601 Campbell Hall, University of California at Berkeley, Berkeley CA 94720-3411, USA

3 National Astronomical Observatory of Japan, Osawa 2-21-1, Mitaka, Tokyo 181-8588, Japan

4 Department of Physics and Astronomy, University College London, Gower Street, London WC1E 6BT, UK

5 Cornell University, Astronomy Department, Ithaca, NY 14853-6801, USA

${ }^{6}$ Research School of Astronomy and Astrophysics, Australian National University, Cotter Road, Weston Creek, ACT 2611, Australia

7 Jodrell Bank Centre for Astrophysics, The University of Manchester, Alan Turing Building, Manchester M13 9PL, UK

Received 23 April 2008 / Accepted 20 June 2008

\section{ABSTRACT}

\begin{abstract}
We present ESO/VLT spectra in the 2.9-4.1 $\mu \mathrm{m}$ range for a large sample of infrared stars in the Small Magellanic Cloud (SMC), mainly carbon stars, massive oxygen-rich Asymptotic Giant Branch (AGB) stars, and red supergiants. Strong emission from Polycyclic Aromatic Hyrdrocarbons (PAHs) is detected in the spectrum of the post-AGB object MSX SMC 29. Water ice is detected in at least one Young Stellar Object, IRAS 01042-7215, for the first time in the SMC. The strength and shapes of the molecular bands detected in the evolved stars are compared with similar data for stars in the Large Magellanic Cloud (LMC). Absorption from acetylene in carbon stars is found to be equally strong in the SMC as in the LMC, but the LMC stars show stronger dust emission in their infrared colours and veiling of the molecular bands. This suggests that a critical link exists in the formation of dust from the molecular atmosphere in carbon stars which scales with the initial metallicity. Nucleation seeds based on a secondary element such as titanium or silicon provide a plausible explanation. In oxygen-rich stars, both the nucleation seeds and molecular condensates depend on secondary elements (in particular titanium, silicon, and/or aluminium), which explains the observed lower molecular abundances and lower dust content in the SMC stars. Emission from silicon monoxide seen in some oxygen-rich AGB stars and red supergiants in the SMC suggests that these metal-poor stars are able to drive strong pulsation shocks through their molecular layers. Data for pulsating dusty AGB stars and supergiants in the LMC are used to show that pulsation is likely the critical factor in driving mass loss, as long as dust forms, rather than the stellar luminosity. Finally, we suggest that the reduced dust production and consequently slower winds of metal-poor AGB stars and red supergiants are more likely to result in chemical inhomogeneities and small-scale structure in the interstellar medium.
\end{abstract}

Key words. stars: carbon - stars: AGB and post-AGB - stars: mass-loss - stars: supergiants - galaxies: Magellanic Clouds - infrared: stars

\section{Introduction}

We owe our existence to the nuclear processing of light elements into heavy elements inside of stars, and their subsequent dispersal into interstellar space by way of stellar mass loss. One of the main contributors of carbon and nitrogen, and arguably the most important "factory" of cosmic dust, Asymptotic Giant Branch (AGB) stars represent the final stages of evolution of intermediate-mass stars $\left(M_{\text {ZAMS }} \lesssim 1\right.$ to $\left.\sim 8 M_{\odot}\right)$ during which they lose up to $\gtrsim 80 \%$ of their mass at rates between $\dot{M} \sim 10^{-6}$ to $10^{-4} M_{\odot} \mathrm{yr}^{-1}$. AGB stars thus chemically enrich the local interstellar medium (ISM) on timescales ranging from less than the dynamical timescale of a galaxy to as long as the age of the Universe. Massive stars $\left(M_{\text {ZAMS }} \gtrsim 8 M_{\odot}\right)$ may become red supergiants (RSGs) and experience similar dusty mass loss too; this greatly affects the properties of the progenitor and circumstellar environment of the subsequent core-collapse supernova, and makes them an important source of dust produced in starbursts observed at cosmological distances.

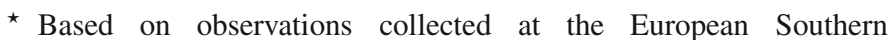
Observatory, Chile (ESO No. 75.D-0696 and 77.D-0614).
Mass loss from red (super)giants happens when strong radial pulsations elevate the stellar atmosphere to a height where the temperature is sufficiently low, but the density is still high, for dust formation to occur. Radiation pressure from these luminous stars $\left(L \sim 5 \times 10^{3}-5 \times 10^{5} L_{\odot}\right)$ is believed to drive away the dust, dragging the gas along with it (e.g., Bowen 1988; Fleischer et al. 1992; Höfner et al. 1995), although the threshold luminosity for this to occur in oxygen-rich environments that produce relatively transparent grains (Ferrarotti \& Gail 2006, and references therein) may be uncomfortably high to explain the mass loss from most oxygen-rich AGB stars (Woitke 2006). At the height of their mass loss dust-enshrouded AGB stars - and in extreme cases even RSGs - vanish at optical wavelengths but shine brightly in the infrared (IR).

An unsolved problem of red (super)giant mass loss is how the simple molecules in the stellar photosphere grow into larger assemblies that form the cores for dust growth (scenarios have been proposed by, e.g., Gail \& Sedlmayr 1988), and how efficient these processes are at the low metallicity that is characteristic for the early Universe. Little is known about the molecular abundances within the dust-formation zone and the dust-to-gas 
ratio in the stellar wind. The transformation of some oxygen-rich AGB stars into carbon stars is known to have extremely important consequences: the molecular and dust formation chemistry will change from oxygen-dominated to carbon-dominated, giving rise to a vastly different array of molecules and dust particles. Hence these two types of AGB star enrich the ISM with very different material. On the other hand, to solve the opacity problem to drive oxygen-rich winds, Höfner \& Andersen (2007) proposed that even oxygen-rich giants may form some carbonaceous grains.

Much of what we know about the mass loss from AGB stars and RSGs has been based on IR observations of dust-enshrouded AGB stars in the Large Magellanic Cloud (LMC, at $50 \mathrm{kpc}$ ) and Small Magellanic Cloud (SMC, at $60 \mathrm{kpc}$ ), where accurate luminosities and mass-loss rates can be obtained (e.g., Wood et al. 1992; Zijlstra et al. 2006; van Loon et al. 1999b, 2005a; van Loon 2000; Matsuura et al. 2005, 2006; Marshall et al. 2004; Sloan et al. 2006; Groenewegen et al. 2007; Lagadec et al. 2007). The Magellanic environments are metal-poor compared to the Sun; their ISM and star clusters (except the oldest ones) have metallicities of typically $[\mathrm{Fe} / \mathrm{H}] \sim 40 \%$ in the LMC and $\sim 15 \%$ in the SMC (e.g., Westerlund 1997; van Loon et al. 2005b).

The above studies suggest that metal-poor carbon stars are surprisingly abundant in $\mathrm{C}_{2}$ and $\mathrm{C}_{2} \mathrm{H}_{2}$, probably because they reach higher $\mathrm{C} / \mathrm{O}$ ratios than their Galactic solar-metallicity equivalents. This may be due to the oxygen-poor photospheres of metal-poor carbon stars, rather than a larger production of primary carbon. This could have important consequences for the condensation of carbonaceous dust grains in low-metallicity environments (cf. Mattsson et al. 2008) - which might however depend on seeds sensitive to initial metallicity such as $\mathrm{TiC}_{2}$ (Bernatowicz et al. 1991). It is as yet unknown how the molecular abundances depend on metallicity in oxygen-rich stars, but here too the nucleation seeds for dust condensation may be restricted to species such as $\mathrm{TiO}$ and $\mathrm{ZrO}$, which are then coated, first by aluminium-oxides and then by silicates (Vollmer et al. 2006; Nittler et al. 2008); this reliance on elements which are not synthesized inside the stars themselves ( $\mathrm{Ti}, \mathrm{Al}, \mathrm{Si}$ ) is consistent with the observed initial-metallicity dependence of oxygen-rich outflows (van Loon 2006; Marshall et al. 2004).

Until now, 3-4 $\mu \mathrm{m}$ spectra of stars in the SMC were limited to two carbon stars published by van Loon et al. (1999a), and another carbon star and one post-AGB object published by Matsuura et al. (2005). Here we present ESO/VLT 3-4 $\mu \mathrm{m}$ spectra of a large sample of dust-enshrouded carbon stars, oxygenrich AGB stars and red supergiants in the SMC, an analysis of their molecular atmospheres and a comparison with previous LMC samples (Matsuura et al. 2005; van Loon et al. 2006). We also present spectra of two R CrB type stars, a post-AGB object showing emission from Polycyclic Aromatic Hydrocarbons (PAHs), and the first 3-4 $\mu \mathrm{m}$ spectra of Young Stellar Objects (YSOs) in the SMC showing water ice absorption and hydrogen recombination lines.

\section{Observations}

\subsection{Target selection}

The targets were selected from IRAS sources (Loup et al. 1997; Groenewegen \& Blommaert 1998), sources observed with ISO (Groenewegen et al. 2000; Cioni et al. 2003), and Spitzer IRS targets (Kraemer et al. 2005, 2006; Sloan et al. 2006; Lagadec et al. 2007; Sloan et al. 2008). These include six stars in the intermediate-age populous star cluster NGC 419, of which the four bluest and least dusty stars would probably not have been selected had they not been part of the cluster sample of Spitzer targets. Of these 76 targets, 57 were actually observed by us this includes one carbon star and one candidate post-AGB object published by Matsuura et al. (2005). The spectroscopic sample thus comprises 36 carbon stars, 14 oxygen-rich AGB stars or RSGs, and 7 peculiar sources (two R CrB-type stars, two post-AGB objects and three candidate YSOs). They are listed in Tables $1-3$.

\subsection{Spectroscopy}

The ISAAC instrument on the European Southern Observatory (ESO) Very Large Telescope (VLT) "Antu" at Paranal, Chile, was used on the nights of 19 to 22 September 2005 and 25 to 28 August 2006 to obtain low-resolution long-slit spectra between 2.9 and $4.1 \mu \mathrm{m}$. At a slit width of $2^{\prime \prime}$ the spectral resolving power was essentially determined by the seeing and was $200 \lesssim$ $R \lesssim 400$. The smallest attained resolution element is sampled by three pixels on the Aladdin array.

The spectra were obtained by chopping and nodding with a throw of $10^{\prime \prime}$ to cancel the high background at these thermalIR wavelengths, and jittering within $2^{\prime \prime}$ to remove the effects of bad pixels. This produced three spectra on the final combined frame, with the central spectrum twice as bright as the other (inverse) spectra. Exposure times varied between 12 and 60 min. After removing cosmic ray hits and correcting the spectra for their small inclination with respect to the array, all spectra were extracted using an optimal extraction algorithm. The extracted spectra were then aligned and combined. Wavelengths calibration was performed with respect to the many telluric absorption lines in the spectra; the spectra are sampled on a grid of $14-\AA$ elements.

The six targets in the cluster NGC 419 were observed pairwise simultaneously, placing both on the slit. The chop/nod throw was enlarged to respectively $13^{\prime \prime}$ for NGC 419IR2 and NGC 419LE 35, which are 8" apart, and 15" for NGC 419LE 27 and NGC 419 LE 18, which are 20" apart. In the latter observation, also light from NGC 419 IR1 was caught in the slit, and its spectrum is combined with that obtained during its dedicated observation through an exposure time-weighted average.

Targets MSX SMC 79 and PMMR 41 were observed using chop/nod throws of $12^{\prime \prime}$ and $15^{\prime \prime}$, respectively, to facilitate the simultaneous observation of an additional star on the slit. One star, IRAS 00454-7257 was observed in 2005, and again in 2006 when it could be placed on the slit and observed simultaneously with another nearby target.

To cancel the telluric absorption and correct for the overall spectral response in the target stars' spectra, a variety of telluric standard stars were observed. These were generally main sequence B stars, but we accidentally also observed two B-type emission-line stars (Appendix A). The imprints left by the hydrogen absorption lines in the quotient spectra of target and standard star are at a level of a few per cent. An example of the telluric absorption affecting the spectra before correction is given in van Loon et al. (2006).

Residuals from telluric line removal and/or bad pixels were suppressed by applying a running boxcar filter with a width of 100 pixels $(0.14 \mu \mathrm{m})$, replacing data values more deviant than three times the median absolute deviation as well as more than $10 \%$ of the mid-spectrum intensity level. The result was inspected visually, and higher thresholds were set in some cases 
Table 1. List of carbon stars, in order of increasing Right Ascension (2MASS coordinates, in J2000). S[..] identifiers are from Groenewegen \& Blommaert (1998), ISO-MCMS J...] identifiers are from Cioni et al. (2003), and NGC 419 LE[..] identifiers are from Lloyd Evans (1980). Near-IR magnitudes are from the 2MASS $\left(J H K_{\mathrm{s}}\right)$ and the spectroscopy acquisition images $\left(L_{\mathrm{NB}}^{\prime}\right)$.

\begin{tabular}{|c|c|c|c|c|c|c|c|c|c|}
\hline Name & Alternative(s) & $\alpha(\mathrm{h} \mathrm{m} \mathrm{s})$ & $\delta\left({ }^{\circ},{ }^{\prime \prime}\right)$ & Date & $J$ & $H$ & $K_{\mathrm{s}}$ & $L_{\mathrm{NB}}^{\prime}$ & Note \\
\hline IRAS 00271-7120 & S1 & 02919.2 & -710350 & $21 / 9 / 05$ & $>17.6$ & $>16.2$ & 14.02 & 8.80 & $\mathrm{a}$ \\
\hline GM 780 & & 03537.3 & -730956 & 19/9/05 & 12.90 & 11.38 & 10.25 & 8.70 & $\mathrm{c}$ \\
\hline IRAS 00388-7401 & S25 & 04043.9 & -734524 & $22 / 9 / 05$ & 15.83 & 13.54 & 11.46 & 9.01 & $\mathrm{a}$ \\
\hline IRAS 00393-7326 & S4 & 04114.4 & -731009 & $20 / 9 / 05$ & 16.26 & 13.97 & 11.97 & 9.40 & $\mathrm{a}$ \\
\hline MSX SMC 54 & & 04305.9 & -732141 & $27 / 8 / 06$ & 16.60 & 14.50 & 12.57 & 9.32 & $\mathrm{~b}$ \\
\hline MSX SMC 44 & & 04339.6 & -731458 & $26 / 8 / 06$ & 12.57 & 11.15 & 10.03 & 9.06 & $\mathrm{~b}$ \\
\hline MSX SMC 105 & & 04502.2 & -725224 & $25 / 8 / 06$ & 15.31 & 13.00 & 11.19 & 9.45 & $\mathrm{~b}$ \\
\hline MSX SMC 36 & & 04554.0 & -732341 & 28/8/06 & 15.07 & 13.23 & 11.60 & 10.54 & $\mathrm{~b}$ \\
\hline IRAS 00448-7332 & MSX SMC 60, S6 & 04640.4 & -731647 & 21/9/05 & 15.68 & 13.15 & 11.20 & 8.46 & $a, b$ \\
\hline MSX SMC 33 & & 04705.5 & -732133 & $25 / 8 / 06$ & 13.45 & 11.70 & 10.32 & 8.27 & $\mathrm{~b}$ \\
\hline IRAS 00454-7257 & S7 & 04718.9 & -724034 & $\begin{array}{l}20 / 9 / 05 \\
26 / 8 / 06\end{array}$ & $>13.1$ & 13.59 & 11.65 & $\begin{array}{l}8.01 \\
9.08\end{array}$ & $\mathrm{a}$ \\
\hline IRAS 00468-7341 & S8 & 04835.7 & -741039 & $21 / 9 / 05$ & $>18.4$ & $>16.5$ & 13.90 & 9.96 & $\mathrm{a}$ \\
\hline MSX SMC 66 & & 04852.5 & -730857 & $26 / 8 / 06$ & 14.70 & 12.70 & 11.12 & 8.28 & $b, i$ \\
\hline RAW 631 & MSX SMC 134 & 05044.4 & -723739 & 26/8/06 & 13.02 & 11.82 & 11.22 & 9.72 & $b, g$ \\
\hline MSX SMC 163 & & 05100.8 & -722519 & $25 / 8 / 06$ & 14.74 & 12.70 & 10.99 & 9.03 & $\mathrm{~b}$ \\
\hline ISO-MCMS J005149.4-731315 & & 05149.5 & -731316 & $21 / 9 / 05$ & 14.26 & 12.56 & 11.17 & 9.10 & $\mathrm{c}, \mathrm{e}$ \\
\hline MSX SMC 125 & & 05150.2 & -725050 & $25 / 8 / 06$ & 13.49 & 11.89 & 10.46 & 8.24 & $\mathrm{~g}$ \\
\hline GM 106 & & 05304.9 & -730410 & 20/9/05 & 14.07 & 12.69 & 11.51 & 9.49 & $\mathrm{c}, \mathrm{h}$ \\
\hline MSX SMC 159 & & 05422.3 & -724330 & 27/8/06 & 16.05 & 13.75 & 11.71 & 9.19 & $\mathrm{~b}$ \\
\hline LEGC 105 & & 05446.9 & -731338 & $27 / 8 / 06$ & 13.27 & 11.80 & 10.73 & 9.50 & $\mathrm{c}$ \\
\hline ISO-MCMS J005450.7-730607 & & 05450.8 & -730607 & $21 / 9 / 05$ & 14.15 & 12.44 & 11.00 & 8.88 & $\mathrm{c}$ \\
\hline ISO-MCMS J005454.1-730318 & & 05454.1 & -730318 & 20/9/05 & 14.35 & 12.36 & 10.79 & 8.40 & $\mathrm{c}, \mathrm{j}$ \\
\hline MSX SMC 209 & & 05616.4 & -721641 & $26 / 8 / 06$ & 13.77 & 12.01 & 10.61 & 9.30 & $\mathrm{~b}$ \\
\hline IRAS 00554-7351 & S16 & 05704.0 & -733515 & 20/9/05 & 16.39 & 13.56 & 11.41 & 8.82 & $\mathrm{a}, \mathrm{c}$ \\
\hline ISO-MCMS J005720.6-731245 & & 05720.6 & -731246 & $21 / 9 / 05$ & 13.84 & 12.40 & 11.33 & 9.30 & $\mathrm{c}$ \\
\hline IRAS 00557-7309 & S17 & 05727.7 & -725328 & $21 / 9 / 05$ & 15.93 & 13.68 & 11.86 & 8.00 & $\mathrm{a}$ \\
\hline MSX SMC 93 & & 05923.4 & -735601 & $25 / 8 / 06$ & 14.42 & 12.71 & 11.41 & 8.78 & $\mathrm{~b}$ \\
\hline NGC 419 LE 16 & & 10801.1 & -725317 & $22 / 9 / 05$ & 13.23 & 11.87 & 10.84 & 9.71 & $\mathrm{c}, \mathrm{f}$ \\
\hline NGC 419 IR1 & & 10813.0 & -725244 & 22/9/05 & 13.48 & 12.04 & 10.89 & 8.76 & $\mathrm{c}, \mathrm{f}$ \\
\hline NGC 419 IR2 & & 10817.5 & -725310 & $22 / 9 / 05$ & - & - & - & 10.17 & $\mathrm{c}, \mathrm{f}$ \\
\hline NGC 419 LE 35 & & 10817.5 & -725301 & $22 / 9 / 05$ & 12.52 & 11.50 & 10.75 & 10.06 & $\mathrm{c}, \mathrm{f}$ \\
\hline NGC 419 LE 27 & & 10820.7 & -725252 & $22 / 9 / 05$ & 12.76 & 11.66 & 11.00 & 10.01 & $\mathrm{c}, \mathrm{f}$ \\
\hline NGC 419 LE 18 & RAW 1553 & 10825.0 & -725257 & $22 / 9 / 05$ & 12.94 & 11.70 & 11.02 & 10.01 & $\mathrm{c}, \mathrm{f}$ \\
\hline GM 522 & RAW 1559 & 10835.4 & -732607 & $25 / 8 / 06$ & 13.86 & 12.53 & 11.44 & 9.90 & $\mathrm{c}$ \\
\hline IRAS 01091-7320 & S23 & 11032.2 & -730504 & $22 / 9 / 05$ & $>16.7$ & 14.68 & 12.34 & 9.73 & $\mathrm{a}$ \\
\hline IRAS 01210-7125 & $\mathrm{S} 30$ & 12228.3 & -710926 & $24 / 7 / 02$ & 14.91 & 12.95 & 11.20 & $(8.1)$ & $\mathrm{d}$ \\
\hline
\end{tabular}

Notes: $\mathrm{a}=$ observed with ISO (Groenewegen et al. 2000); $\mathrm{b}=$ Spitzer spectrum (Sloan et al. 2006); $\mathrm{c}=$ Spitzer spectrum (Lagadec et al. 2007); $\mathrm{d}=L$-band spectrum published in Matsuura et al. (2005); e = carbon star RAW 704 (BMB-B 70) is at 5" to SE; f = see Tanabé et al. (2004) and van Loon et al. (2005b); $\mathrm{g}=$ Spitzer spectrum (Sloan et al., in prep.); $\mathrm{h}=$ Spitzer spectrum is of PMMR 52 (see Table 4); $\mathrm{i}=$ IRAS $00472-7325$ at $1^{\prime}$ to $\mathrm{SE} ; \mathrm{j}=$ carbon star RAW 892 at $10^{\prime \prime}$ to SE.

to prevent sharp absorption features or emission lines being affected by this procedure.

The observing conditions varied between fair and poor; although the humidity was quite stable around $30 \%$ and $10 \%$ during the 2005 and 2006 runs, respectively, the optical seeing had the tendency of increasing from $\sim 1^{\prime \prime}$ at the start of the night to $\sim 1.5^{\prime \prime}$ in the 2005 run, and to over $2.5^{\prime \prime}$ in the 2006 run (the IR seeing was better). Interruptions occurred due to cloud cover, especially in the 2005 run when clouds would form suddenly upon sunset.

\subsection{Photometry}

IR photometry is compiled for all targets and for the two objects observed by Matsuura et al. (2005), as well as for the serendipitous objects that were placed on the slit whilst observing one of the primary targets (Table 4).
$J H K_{\mathrm{s}}$-band photometry is available from the 2-Micron AllSky Survey (2MASS: Skrutskie et al. 2006), but some of them are too faint in the $J$-band to have been detected in the 2MASS. $L_{\mathrm{NB}}^{\prime}$-band $\left(\lambda_{\text {centre }}=3.80 \mu \mathrm{m}, \Delta \lambda=0.06 \mu \mathrm{m}\right)$ photometry is measured from the spectroscopy acquisition images, calibrated against the acquisition images of the telluric standard stars and generally accurate to within $\sim 0.1 \mathrm{mag}$. Photometry for NGC 419 is also available from van Loon et al. (2005b) - who first detected NGC 419IR2 in the $K$-band (cf. Groenewegen et al. 2007) - and Tanabé et al. (2004).

For IRAS 01210-7125 and IRAS 00350-7436, the spectra of which were published by Matsuura et al. (2005), we measured (broad-band) $L$-band magnitudes from their acquisition images.

Van Loon et al. (2006) and Whitelock et al. (2003) published photometry for sources in the LMC with which we compare our new data, but note that the $L$-band filter used by Whitelock et al. (2003) differs from the 3.80- $\mu \mathrm{m}$ narrowband filter used by us. 
Table 2. List of dust-enshrouded oxygen-rich AGB stars and red supergiants. See the description of Table 1.

\begin{tabular}{|c|c|c|c|c|c|c|c|c|c|c|}
\hline Name & Alternative(s) & $\alpha\left(\mathrm{h} \mathrm{m} \mathrm{s}^{2}\right)$ & $\delta\left({ }^{\circ}, \prime \prime\right)$ & Date & $J$ & $H$ & $K_{\mathrm{s}}$ & $L_{\mathrm{NB}}^{\prime}$ & SpT & Note \\
\hline HV 1375 & MSX SMC 24 & 04252.2 & -735052 & $28 / 8 / 06$ & 11.30 & 10.38 & 9.83 & 8.64 & M5 & $\mathrm{b}$ \\
\hline MSX SMC 18 & & 4631.6 & -732846 & $25 / 8 / 06$ & 12.66 & 11.16 & 10.20 & 8.01 & & $\mathrm{~b}$ \\
\hline BFM 1 & & 04719.2 & -724005 & 26/8/06 & 12.66 & 11.65 & 11.03 & 9.72 & $\mathrm{~S}$ & $\mathrm{c}$ \\
\hline HV 11262 & MSX SMC 67 & 04736.9 & -730444 & $25 / 8 / 06$ & 9.47 & 8.60 & 8.32 & 7.98 & M0.5 & $\mathrm{b}$ \\
\hline PMMR 34 & SMC 96 & 05006.4 & -732811 & 27/8/06 & 9.59 & 8.76 & 8.54 & 8.45 & M0.5 & b \\
\hline IRAS 00483-7347 & S9 & 05007.2 & -733125 & $20 / 9 / 05$ & 11.43 & 9.78 & 8.64 & 6.84 & M8 & $\mathrm{b}$ \\
\hline GM 103 & IRAS $00486-7308$, S 10 & 05030.6 & -725130 & $20 / 9 / 05$ & 10.34 & 9.39 & 8.78 & 8.18 & M4 & $\mathrm{a}, \mathrm{d}$ \\
\hline PMMR 41 & MSX SMC 109 & 05129.7 & -731044 & $27 / 8 / 06$ & 9.22 & 8.41 & 8.16 & 7.81 & M0 Iab & $\mathrm{b}$ \\
\hline BMB-B 75 & & 05212.9 & -730853 & $27 / 8 / 06$ & 11.25 & 10.47 & 9.92 & 8.71 & M & $\mathrm{b}, \mathrm{e}$ \\
\hline HV 1652 & MSX SMC 168 & 05526.8 & -723556 & 28/8/06 & 9.80 & 9.01 & 8.76 & 8.30 & M0.5 & $\mathrm{b}$ \\
\hline HV 11417 & $\begin{array}{l}\text { MSX SMC 149, } \\
\text { IRAS 00591-7307, S18 }\end{array}$ & 10048.2 & -725102 & 21/9/05 & 9.75 & 8.81 & 8.45 & 7.80 & M5 e & $\mathrm{a}, \mathrm{b}$ \\
\hline IRAS 01066-7332 & S20a & & -731552 & $25 / 8 / 06$ & 11.19 & 10.20 & 9.59 & 9.09 & M8 & $a, b$ \\
\hline HV 12956 & IRAS 01074-714 & 10902.3 & -712410 & $22 / 9 / 05$ & 11.53 & 10.85 & 10.34 & 9.15 & M5 & $a, f$ \\
\hline HV 2084 & IRAS 01082-7335, S29 & 10938.2 & -732002 & 28/8/06 & 8.85 & 8.14 & 7.79 & 7.49 & M2 Ia & $\mathrm{b}$ \\
\hline
\end{tabular}

Notes: $\mathrm{a}=$ observed with ISO (Groenewegen et al. 2000); $\mathrm{b}=$ Spitzer spectrum (Sloan et al., in prep.); $\mathrm{c}$ = star of S type (Blanco et al. 1981; Sloan et al. 2008); $\mathrm{d}$ = groundbased mid-IR spectrum (Groenewegen et al. 1995); e = has been confused with a carbon star, a radio continuum source is at $6^{\prime \prime}$ to $W$ (Filipović et al. 2002); f = Li-rich (Smith et al. 1995).

Table 3. List of peculiar objects. See the description of Table 1.

\begin{tabular}{|c|c|c|c|c|c|c|c|c|c|}
\hline Name & Alternative(s) & $\alpha(\mathrm{h} \mathrm{m} \mathrm{s})$ & $\delta\left({ }^{\circ},{ }^{\prime \prime}\right)$ & Date & $J$ & $H$ & $K_{\mathrm{s}}$ & $L_{\mathrm{NB}}^{\prime}$ & Note \\
\hline \multicolumn{10}{|l|}{ RCrB-type stars } \\
\hline MSX SMC 14 & & 04616.3 & -741114 & $27 / 8 / 06$ & $>17.4$ & $>15.8$ & 13.97 & 9.79 & $\mathrm{c}$ \\
\hline MSX SMC 155 & & 05718.2 & -724235 & $25 / 8 / 06$ & 13.88 & 12.81 & 11.73 & 8.51 & $\mathrm{c}$ \\
\hline \multicolumn{10}{|l|}{ Post-AGB (C) objects } \\
\hline MSX SMC 29 & & 03646.3 & -733135 & $26 / 8 / 06$ & $>16.6$ & 15.75 & 13.50 & 10.47 & $\mathrm{~d}$ \\
\hline IRAS 00350-7436 & $\mathrm{S} 2$ & 03659.6 & -741950 & $24 / 7 / 02$ & 11.32 & 10.16 & 9.13 & $(7.7)$ & $\mathrm{e}$ \\
\hline \multicolumn{10}{|c|}{ Candidate Young Stellar Objects } \\
\hline MSX SMC 79 & & 04839.7 & -732501 & $27 / 8 / 06$ & 16.57 & 15.54 & 14.34 & 10.82 & $\mathrm{~b}$ \\
\hline IRAS 01039-7305 & MSX SMC 180, DEM S129 & 10530.3 & -724954 & 28/8/06 & 14.86 & 13.24 & 11.69 & 8.57 & $\mathrm{~b}$ \\
\hline IRAS 01042-7215 & S28 & 10549.3 & -715949 & $26 / 8 / 06$ & 16.52 & 15.15 & 13.29 & 9.24 & $\mathrm{a}, \mathrm{f}$ \\
\hline
\end{tabular}

Notes: a = observed with ISO (Groenewegen et al. 2000); b = Spitzer spectrum (Sloan et al., in prep.); c = Spitzer spectrum (Kraemer et al. 2005); $\mathrm{d}=$ Spitzer spectrum (Kraemer et al. 2006); e = L-band spectrum published by Matsuura et al. (2005); f = Spitzer IRS and MIPS-SED cycle 5 target (P.I. Sloan).

Table 4. Serendipitous objects, listed in order of increasing Right Ascension (2MASS coordinates, in J2000). Near-IR magnitudes are from the 2MASS $\left(J H K_{\mathrm{s}}\right)$ and the spectroscopy acquisition images $\left(L_{\mathrm{NB}}^{\prime}\right)$.

\begin{tabular}{|c|c|c|c|c|c|c|c|c|c|}
\hline Name & $\alpha(\mathrm{h} \mathrm{m} \mathrm{s})$ & $\delta\left({ }^{\circ}, \prime \prime\right)$ & Date & $J$ & $H$ & $K_{\mathrm{s}}$ & $L_{\mathrm{NB}}^{\prime}$ & Type & Note \\
\hline 2MASS J00472001-7240350 & 04720.0 & -724035 & $20 / 9 / 05$ & 11.43 & 10.52 & 10.30 & 10.01 & O-rich & \\
\hline 2MASS J00473917-7304115 & 04739.2 & -730412 & 25/8/06 & 14.58 & 12.97 & 11.56 & 9.55 & C star & \\
\hline MSX SMC 79 B & 04841.0 & -732503 & 27/8/06 & - & - & - & 11.80 & YSO? & \\
\hline RAW 616 & 05022.9 & -725141 & 20/9/05 & 12.91 & 11.88 & 11.38 & 10.53 & Post-AGB (C) object? & \\
\hline RAW 639 & 05050.6 & -723719 & 26/8/06 & 12.87 & 11.74 & 11.19 & 10.46 & C star & \\
\hline 2MASS J00513146-7310513 & 05131.5 & -731051 & 27/8/06 & 10.85 & 10.10 & 9.86 & 9.59 & O-rich & \\
\hline PMMR 52 & 05309.1 & -730404 & 20/9/05 & 9.26 & 8.45 & 8.22 & 7.90 & K5 & a \\
\hline 2MASS J00544039-7313407 & 05440.4 & -731341 & $27 / 8 / 06$ & 11.77 & 10.91 & 10.60 & 10.20 & O-rich & \\
\hline PMMR 79? & 05536.6 & -723624 & 28/8/06 & 9.88 & 9.12 & 8.91 & 8.54 & K5 & \\
\hline 2MASS J00565642-7335301 & 05656.4 & -733530 & 20/9/05 & 14.94 & 13.22 & 11.66 & 10.32 & C star & \\
\hline RAW 1030 & 05711.6 & -731315 & 21/9/05 & 12.77 & 11.81 & 11.37 & 10.89 & C star? & \\
\hline PMMR 116 & 10054.2 & -725137 & 21/9/05 & 9.52 & 8.92 & 8.68 & 8.58 & K6.5 & \\
\hline
\end{tabular}

Notes: $\mathrm{a}=$ Spitzer spectrum (Lagadec et al. 2007).

\section{Results}

\subsection{Global properties of the sample}

The following subsections describe the spectroscopic identification of the various types of objects; the results are used already here to describe their photometric properties.
All our targets have $K_{\mathrm{s}}$-band and $3.80-\mu \mathrm{m}$ photometry; the corresponding colour-magnitude diagram is displayed in Fig. 1, for all but the NGC 419 stars which are displayed in Fig. 2 instead. The acquisition images sample stars brighter than the tip of the RGB. Stars that were not targets for our spectroscopy ("field") lie on the main AGB sequence $\left(K_{\mathrm{S}}-L_{\mathrm{NB}}^{\prime} \lesssim 1\right.$ mag and $M_{K \mathrm{~s}} \gtrsim-9 \mathrm{mag}$ ); these include the many optically bright carbon 


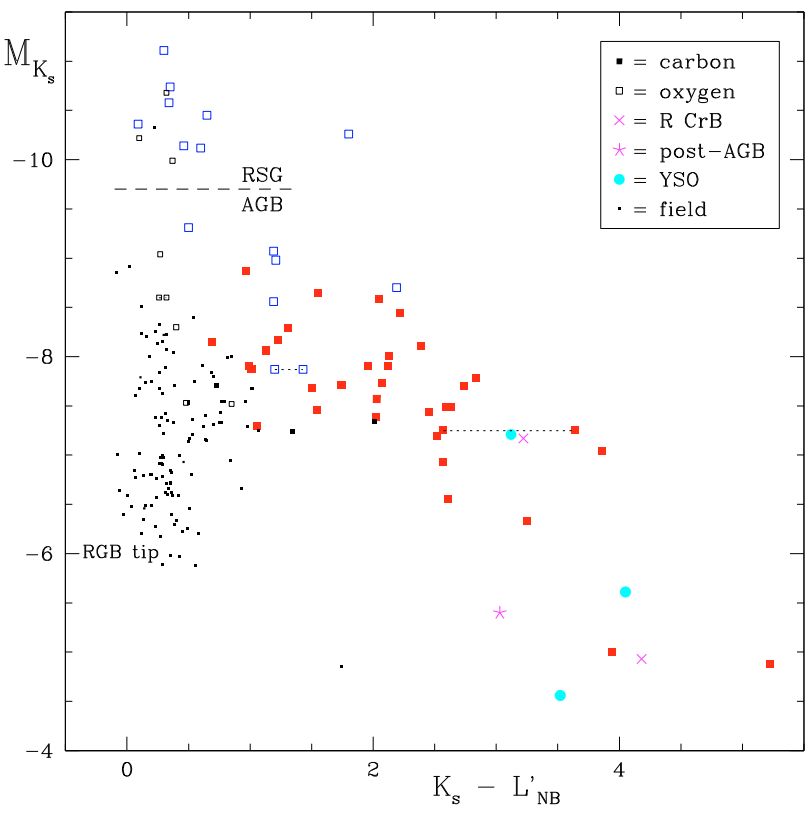

Fig. 1. 2MASS-ISAAC colour-magnitude diagram of targets and field stars, except the objects in NGC 419 (see Fig. 2). Serendipitously observed stars are indicated with smaller squares (in black) than the primary targets (in blue and red).

stars known in the SMC (e.g., Rebeirot et al. 1993), that have little dust and are therefore less interesting for the purpose of this study and often rather faint for 3-4 $\mu \mathrm{m}$ spectroscopy. Our targets are brighter in $L_{\mathrm{NB}}^{\prime}$.

There is a clear gap in $K_{\mathrm{s}}$-band magnitude between the brightest AGB stars (which tend to be oxygen-rich) and the RSGs. This may correspond to the realm of the super-AGB stars, with masses around $8 M_{\odot}$ or so, which are found to become less cool and dusty than AGB stars or slightly more massive RSGs (van Loon et al. 2005a,b). Photometric variability and overluminosity as a result of Hot Bottom Burning (HBB; Boothroyd \& Sackmann 1992) could cause an AGB star to be classified as a RSG - HV 11417 may be an example of this, as it has a large $K$-band amplitude of $\Delta K=1.3 \mathrm{mag}$ which is uncommon for stars as luminous as RSGs, and the 2MASS values correspond to near-maximum light.

Carbon stars start digressing from the vertical AGB sequence around $M_{\mathrm{Ks}} \simeq-7.5 \mathrm{mag}$, towards redder colours. They quickly become dusty; stars redder than the vertical AGB sequence by $\Delta\left(K_{\mathrm{s}}-L_{\mathrm{NB}}^{\prime}\right) \sim 0.7 \mathrm{mag}$ are almost invariably spectroscopic targets. Oxygen-rich AGB stars bright enough for our spectroscopic programme are generally brighter (and presumably more massive) than the carbon stars. Although not a carbon star, BFM 1 sits at a locus in the colour-magnitude diagram that is otherwise dominated by carbon stars. This star is classified as an S-type star with a carbon-to-oxygen ratio nearly 1 by Blanco et al. (1981), which was confirmed by the detection of $\mathrm{LaO}$ in its optical spectrum by Sloan et al. (2008). It is a Mira-type variable (with a 394 days optical period) and $L_{\mathrm{NB}}^{\prime}=9.60$ and $9.83 \mathrm{mag}$ in 2005 and 2006, respectively.

Rare phases of extreme dust obscuration are seen in the form of a few carbon stars with $K_{\mathrm{s}}-L_{\mathrm{NB}}^{\prime}>3 \mathrm{mag}$. The fact that no such obscured oxygen-rich AGB stars are present in our sample (whereas many Galactic examples are known; e.g., Jones \& Merrill 1976) may be due to the following effects: (1) oxygenrich dust is more transparent at near-IR wavelengths, especially if the iron content is low (Woitke 2006) which may be the case in

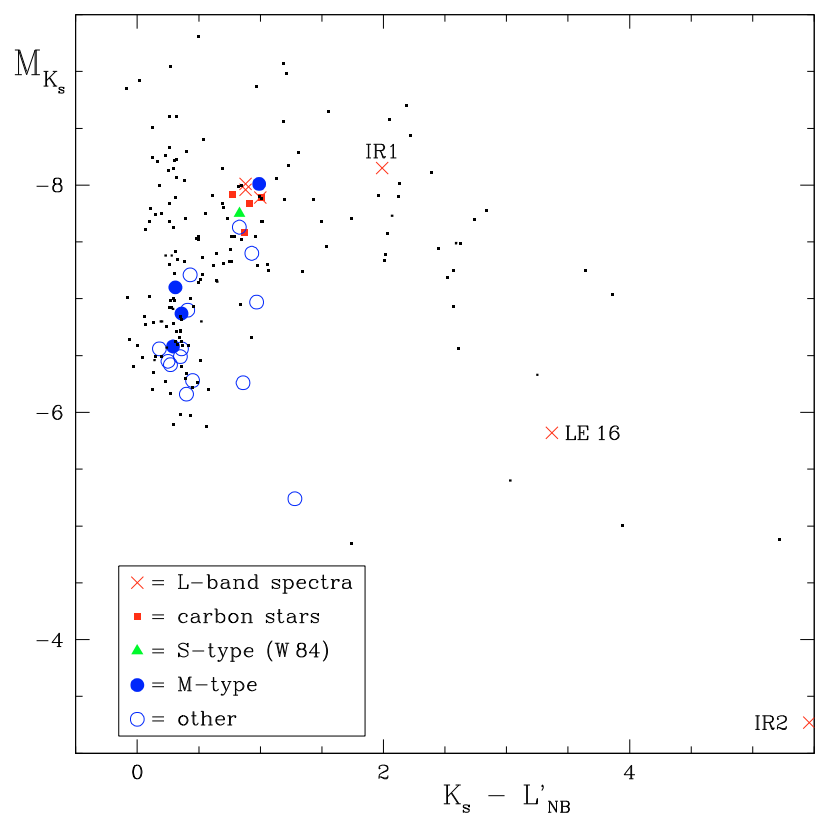

Fig. 2. SOFI-ISAAC colour-magnitude diagram for the intermediateage populous cluster NGC 419. The stars from Fig. 1 (excluding the $\mathrm{R} \mathrm{CrB}$ and YSO objects and field stars) are plotted as small dots for reference. All 6 targets for which $L$-band spectra were obtained (crosses) are carbon stars.

the metal-poor SMC, which together with the generally low dust content in the SMC renders these stars less obscured; (2) more luminous AGB stars have more extended and hence more diluted circumstellar envelopes (the optical depth scales roughly as $\tau \propto r^{-1} \propto L^{-0.5}$, where $r$ is the inner radius of the dust envelope) - in this regard, IRAS 00483-7347 is an extreme example of a dust-enshrouded RSG; (3) the initial mass function (IMF) and rapid evolution of relatively massive stars conspire to make it difficult to catch such a star in the most dust-enshrouded phase, especially in a small galaxy such as the SMC; (4) carbon stars form at lower masses in the Magellanic Clouds than in the Milky Way (e.g., Marigo et al. 1999), thereby enlarging the population of carbon stars at the expense of low-mass oxygen-rich AGB stars.

The two RCrB-type stars and post-AGB object MSX SMC 29 are amongst the reddest objects, and fainter than the bulk of the carbon stars. The reason for the faintness may in part be due to their hotter stellar photospheric temperatures (hence larger IR bolometric corrections), not just dust obscuration. In that regard, candidate post-AGB object IRAS 00350-7436 (Whitelock et al. 1989; Matsuura et al. 2005) is rather bright in $K$.

Three very red objects show the $3-\mu \mathrm{m}$ water ice feature and/or hydrogen emission lines, which suggest these are hot stars embedded in thick, cold dust cocoons and most likely YSOs. Although some Galactic AGB/post-AGB stars are known to exhibit the water ice feature (e.g., Smith et al. 1988), this is not commonly observed; the location of these three objects in obvious star-forming regions makes an identification as YSOs more plausible. One field star, 2MASS J00462938-7315530 is quite red $\left(J-K_{\mathrm{s}}=2.0 \mathrm{mag}, K_{\mathrm{S}}-L_{\mathrm{NB}}^{\prime}=1.7 \mathrm{mag}\right)$ and very faint. It is seen in the direction of Dark Nebula \#5 identified by Hodge (1974), and it might well be a star behind this dust cloud or a YSO within it. 


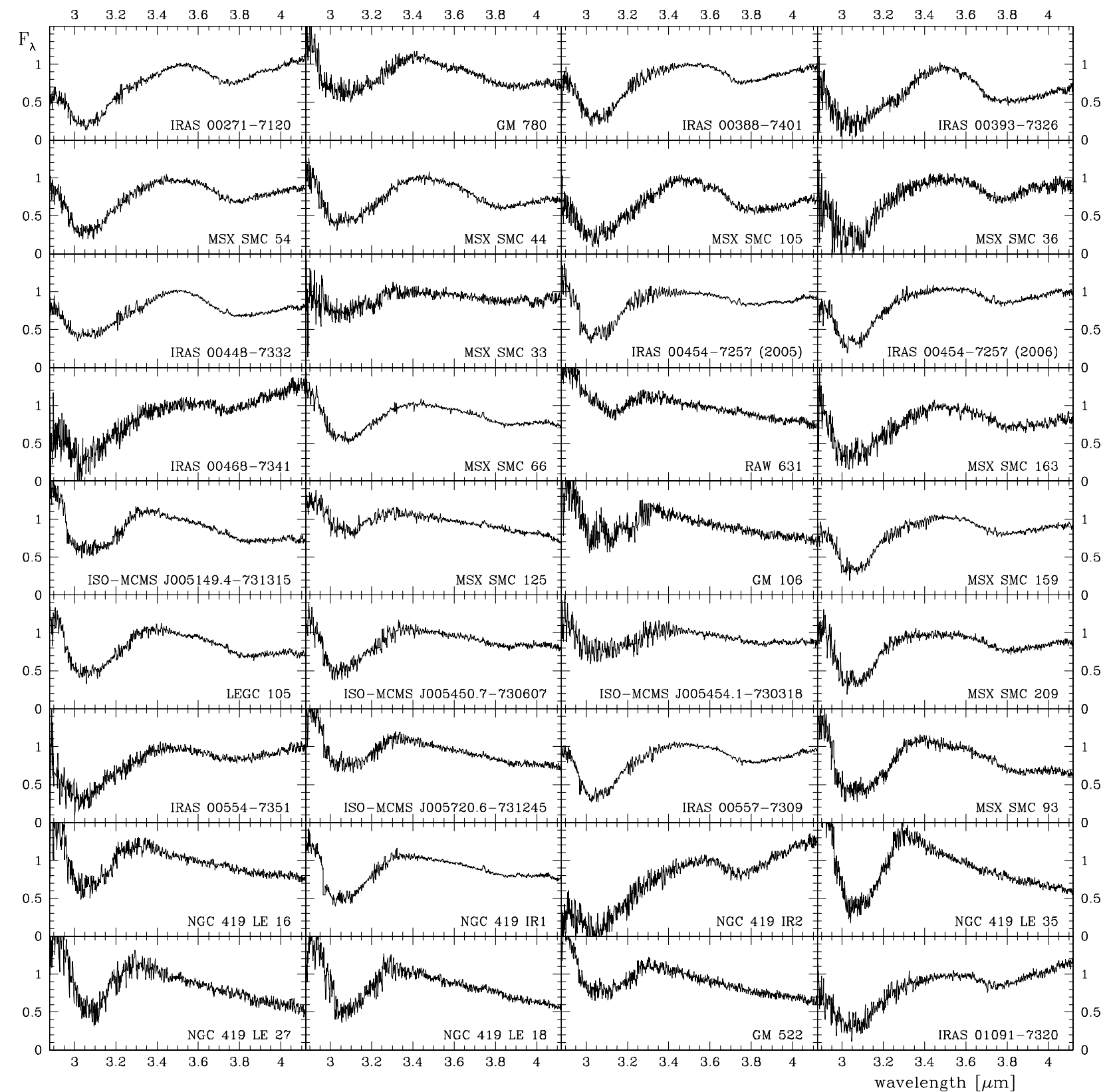

Fig. 3. VLT/ISAAC 3-4 $\mu \mathrm{m}$ spectra of IR-bright carbon stars in the SMC. The most conspicuous features are the broad absorption bands of acetylene $\left(\mathrm{C}_{2} \mathrm{H}_{2}\right)$ and hydrogen cyanide $(\mathrm{HCN})$ around $3.1 \mu \mathrm{m}$ and often also around $3.8 \mu \mathrm{m}$.

\subsubsection{Dusty stars in the populous cluster NGC 419}

The stars in NGC 419 follow the trends outlined above (Fig. 2). All stars for which we obtained $L$-band spectra are carbon stars, and they are indeed where IR-bright carbon stars are expected.

NGC 419 IR2 (Tanabé et al. 1999; van Loon et al. 2005b) is the most extremely dust-enshrouded carbon star known in the SMC, but two more carbon stars in NGC 419, LE 16 (Lloyd Evans 1980) and IR1 (Tanabé et al. 1997; van Loon et al. 2005 b) are also very dusty. LE 16 is a large-amplitude variable: the $K_{\mathrm{s}}$-band magnitude from the 2MASS is $10.84 \mathrm{mag}$ whilst it is $13.08 \mathrm{mag}$ on our SOFI images (van Loon et al. 2005 b). IR 2 was detected in the near-IR only on our SOFI images $\left(K_{\mathrm{s}}=15.63 \mathrm{mag}\right)$ and by Groenewegen et al. (2007).

The three other carbon stars for which we obtained $L$-band spectra are not very red, and occupy the locus in the colour-magnitude diagram where also an M-type (Lloyd Evans 1983) and an S-type star (W 84, Walker 1972) are known to exist in NGC 419. Three further confirmed M-type stars (Lloyd Evans 1983) in NGC 419 occupy the lower portion of the vertical AGB sequence. A few other stars in NGC 419 are clearly redder than the main AGB sequence, but fainter than the red carbon star branch. It is unclear what causes their red colours, but interstellar extinction is a possibility.

\subsection{Spectra of carbon stars}

The carbon stars are listed in Table 1, and their spectra are displayed in Fig. 3 (normalised to a flux density of 1 at $3.5 \mu \mathrm{m}$ ). They all show the prominent absorption band around $3.1 \mu \mathrm{m}$ due to the toxic gases acetylene $\left(\mathrm{C}_{2} \mathrm{H}_{2}\right)$ and hydrogen cyanide $(\mathrm{HCN})$, and in most cases also the $3.8 \mu \mathrm{m}$ acetylene 


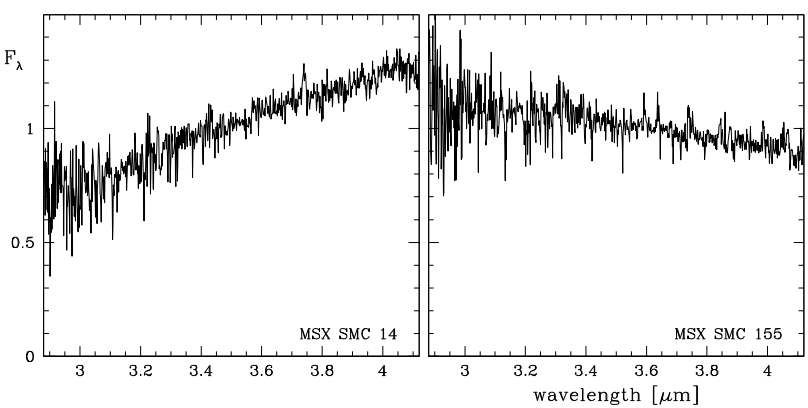

Fig. 4. VLT/ISAAC 3-4 $\mu$ m spectra of two R CrB type stars in the SMC, displaying a featureless dust emission continuum.

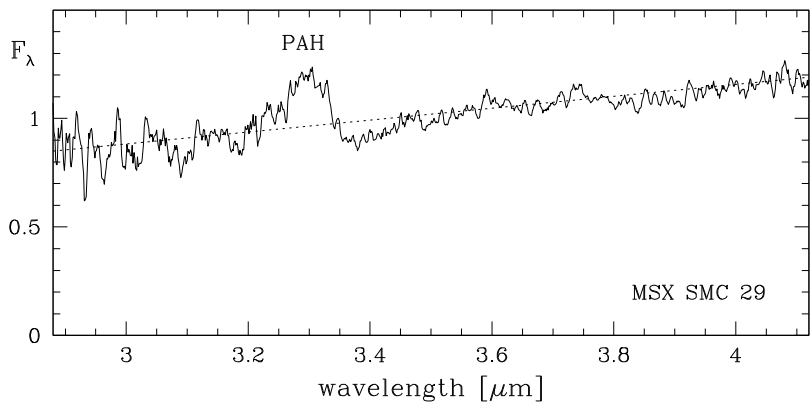

Fig. 5. VLT/ISAAC $3-4 \mu \mathrm{m}$ spectrum of a post-AGB object in the SMC, displaying a dust emission continuum as well as a prominent emission band at $3.28 \mu \mathrm{m}$ attributed to Polycyclic Aromatic Hydrocarbons (PAHs).

band. The much sharper $3.57 \mu \mathrm{m}$ HCN band is visible in some stars, but only weakly (e.g., IRAS 00393-7326). The band strengths and shapes and continuum slopes are discussed in Sect. 4.1.

GM 106 was observed during poor conditions, and its $3.1-\mu \mathrm{m}$ band is clearly affected by the weak signal below $3.4 \mu \mathrm{m}$. IRAS 00393-7326 and IRAS 00554-7351 were the first SMC stars ever for which an $L$-band spectrum was published (van Loon et al. 1999a), but our new spectra are of a superior quality, resolution and spectral coverage.

\subsection{Spectra of R CrB-type stars}

Two RCrB-type stars were observed with the Spitzer IRS (Kraemer et al. 2005); they are listed in Table 3 and their $L$-band spectra are displayed in Fig. 4. These are carbon-rich objects which are dominated in this wavelength region by a dust emission continuum (e.g., Lambert et al. 2001). It is clear both from the photometry and the spectra that MSX SMC 14 is dustier than MSX SMC 155 (cf. Kraemer et al. 2005). An optical spectrum of MSX SMC 155 reveals it to be a rather cool carbon star, suggesting that it is still near the AGB (Kraemer et al. 2005).

\subsection{Spectra of post-AGB objects}

A post-AGB object observed with the Spitzer IRS (Kraemer et al. 2006) is listed in Table 3; its $L$-band spectrum is displayed in Fig. 5. This object exhibits a prominent emission band around $3.28 \mu \mathrm{m}$, which is attributed to PAHs. It also shows a hint of absorption at $3.4 \mu \mathrm{m}$, which we discuss in Sect. 4.1.4. Another example of such an object, the $L$-band spectrum of IRAS 00350-7436 was published by Matsuura et al. (2005);

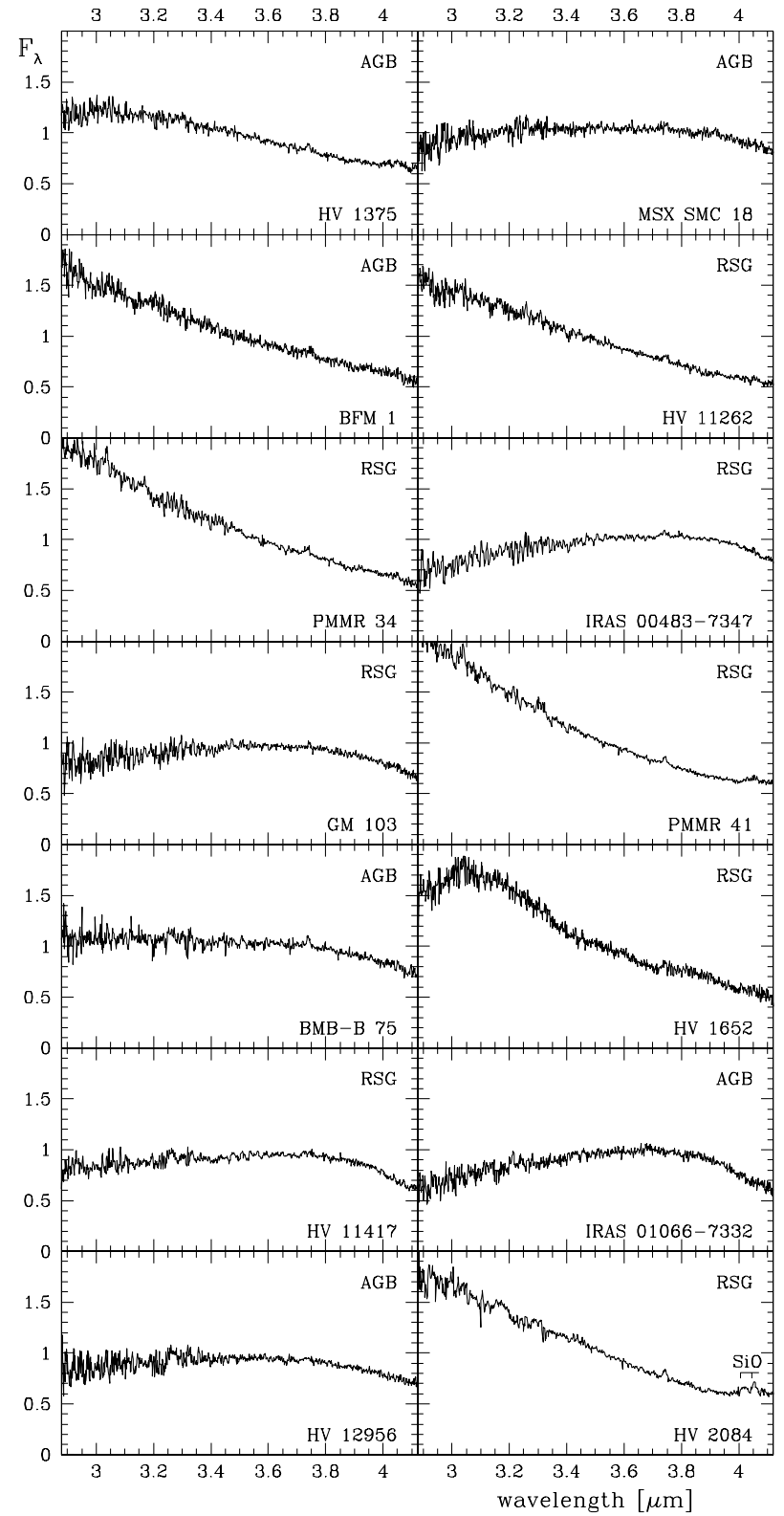

Fig. 6. VLT/ISAAC 3-4 $\mu \mathrm{m}$ spectra of oxygen-rich AGB stars and red supergiants in the SMC. The spectral curvature is determined mostly by the strength of dust emission and water vapour absorption; in some stars the silicon monoxide ( $\mathrm{SiO}$ ) band around $4 \mu \mathrm{m}$ is seen, either in absorption or in emission.

it is listed in Table 3 for completeness. The presence of PAHs suggests that these post-AGB objects descended from carbon stars.

\subsection{Spectra of oxygen-rich AGB stars and RSGs}

A collection of oxygen-rich stars were observed (Table 2; Fig. 6). As discussed in Sect. 3.1 these separate out into AGB stars and RSGs on the basis of their $K$-band luminosity. Although there are no clear-cut differences in spectral appearance between these two classes, RSGs do seem to show the first overtone $\mathrm{SiO}$ band at $4 \mu \mathrm{m}$ more often in emission. The best examples of the latter are also the most luminous in the sample, viz. PMMR 41 and HV 2084. The $\mathrm{SiO}$ band is discussed in more detail in Sect. 4.2. 


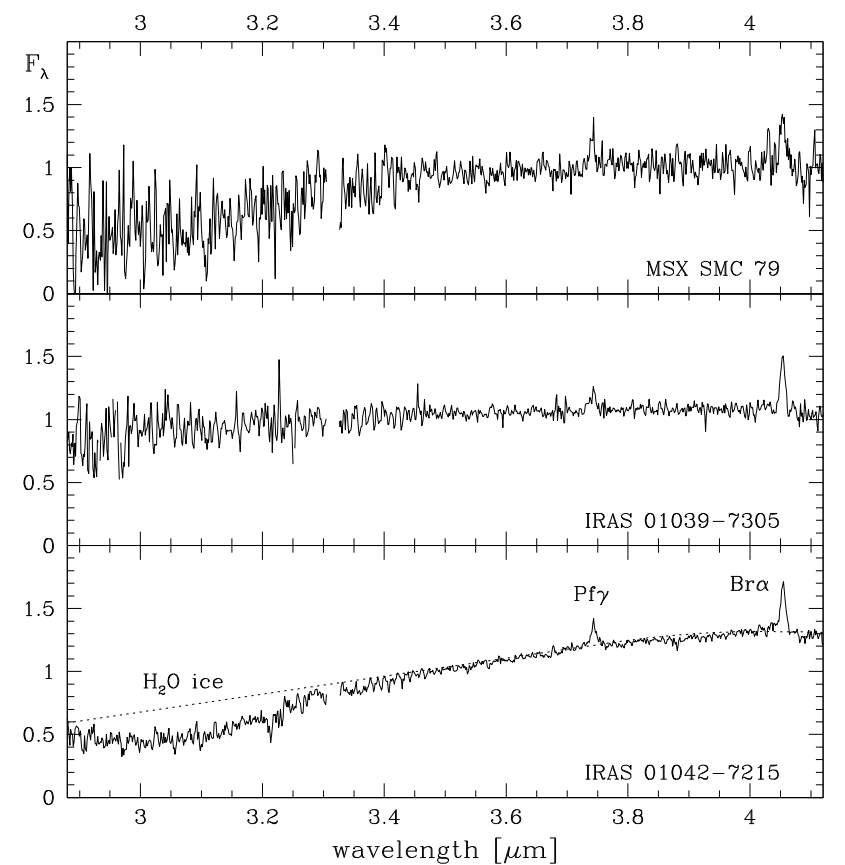

Fig. 7. VLT/ISAAC 3-4 $\mu \mathrm{m}$ spectra of YSO candidates in the SMC. All three show hydrogen line emission, and water ice absorption around $3.1 \mu \mathrm{m}$ is prominent in the reddest of them, IRAS 01042-7215 (the dotted curve is a 2nd-order polynomial fit to the continuum beyond $3.6 \mu \mathrm{m})$.

The spectrum of the S-type star BFM 1 is rather featureless and not very red, indicating little molecular absorption or dust emission. This is commensurate with its not very red colours.

BMB-B 75 is listed in Simbad and recent papers as a carbon star, but it was discovered as an oxygen-rich AGB star of spectral type M6 (Blanco et al. 1980). The latter is consistent with our spectrum, with the Spitzer spectrum showing silicate emission (Sloan et al., in prep.), and with the long pulsation period of 1453 days (Cioni et al. 2003).

HV 1652 shows strong absorption shortward of $3 \mu \mathrm{m}$, possibly water vapour. There is also a broad depression running from about 3.4 until $3.75 \mu \mathrm{m}$ - possibly due to $\mathrm{OH}$ (Wallace \& Hinkle 2002).

\subsection{Spectra of candidate Young Stellar Objects}

Three of the oxygen-rich objects show hydrogen lines in emission, and at least in one case (IRAS 01042-7215, the reddest of the three) absorption in the $3-\mu \mathrm{m}$ band of water ice is prominent (Fig. 7; Table 3). MSX SMC 79 is situated near the young star cluster Bruck 48. These are good candidates for being massive embedded Young Stellar Objects (YSOs), and the first such examples in the SMC for which $L$-band spectra are presented.

It is interesting to note that in contrast to the first massive YSO studied in this way in the LMC, IRAS 05328-6827 (van Loon et al. 2005c), there is very little trace of methanol ice $\left(\mathrm{CH}_{3} \mathrm{OH}\right)$ in the spectrum of IRAS 01042-7215 whilst the water ice band is stronger than in the LMC object, viz. $N\left(\mathrm{H}_{2} \mathrm{O}\right) \sim$ $4 \times 10^{17} \mathrm{~cm}^{-2}$. This might reflect a lower efficiency of ice processing as a result of a reduced total grain surface area and/or a reduced interstellar abundance of carbon monoxide. Oliveira et al. (2008) present a much more detailed study of the chemistry in massive YSOs in the SMC, and Oliveira et al. (2006) presented more LMC examples.

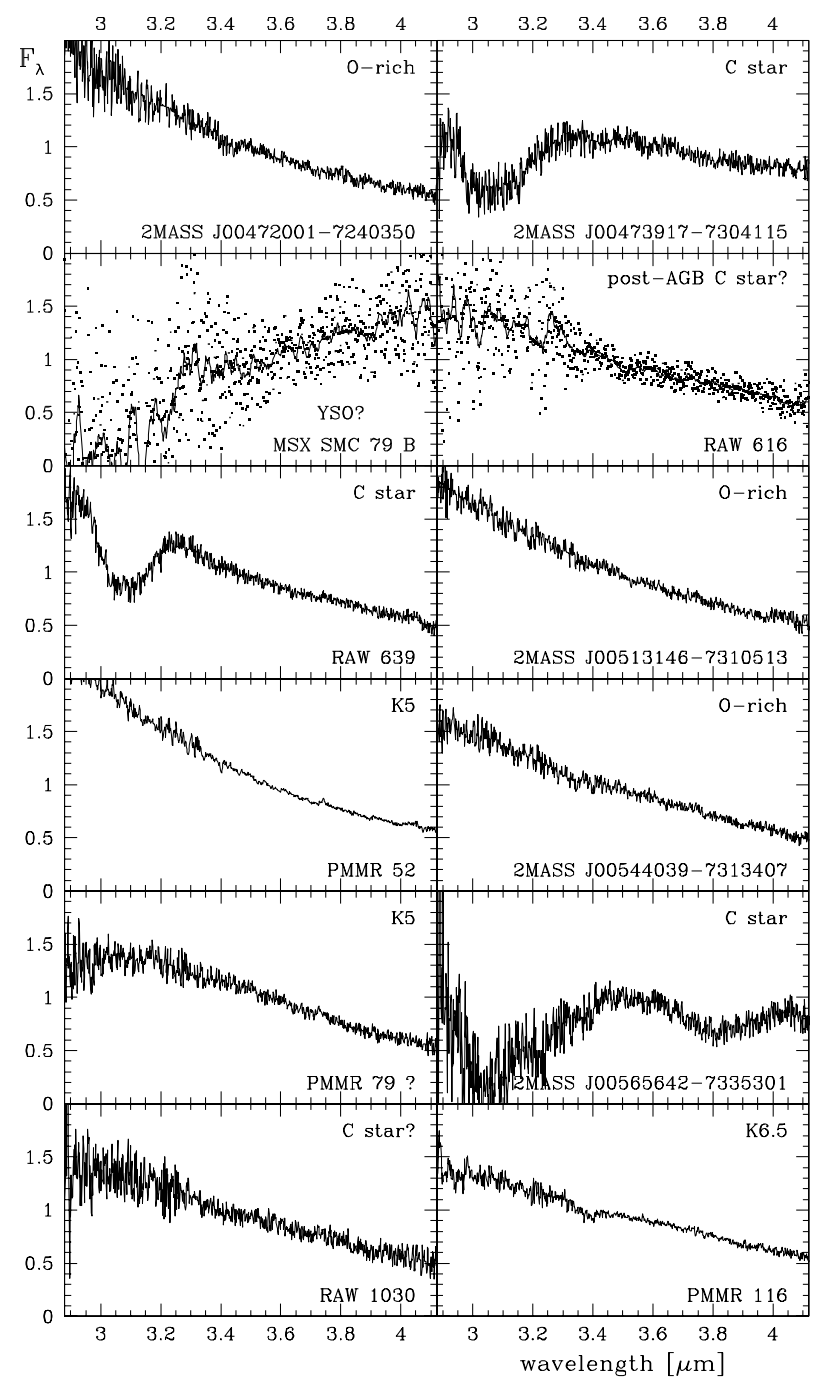

Fig. 8. VLT/ISAAC 3-4 $\mu \mathrm{m}$ spectra of stars in the SMC that were observed simultaneously with primary targets. These objects comprise both carbon stars and oxygen-rich giants, and possibly an embedded YSO (MSX SMC 79 B) and a post-AGB object (RAW 616) - the latter two were heavily smoothed but the original spectral data are also plotted (as dots).

\subsection{Serendipitous spectra}

Red and/or IR-bright objects were sometimes identified and placed on the slit to be observed simultaneously with a primary target (Table 4 and Fig. 8).

Three are carbon stars: 2MASS J00473917-7304115, RAW 639 and 2MASS J00565642-7335301. Whereas the first two are fairly warm the other is cool and dusty and shows strong absorption in the 3.8- $\mu \mathrm{m}$ band of acetylene. Two more stars are believed to be carbon stars, RAW 616 and RAW 1030, but their spectra show no trace of the $3-\mu \mathrm{m}$ band. Veiling by dust emission cannot be the explanation in this case as these two stars are not very dusty at all. Late-R-type carbon stars also do not always show the 3- $\mu \mathrm{m}$ band (e.g., Le Bertre et al. 2005). After heavily smoothing the spectrum, RAW 616 shows a hint of the $3.28-\mu \mathrm{m}$ PAH band in emission, which means that it might be a carbon star in its post-AGB phase. This is rather unlikely to have been discovered serendipitously, and it is possible that the PAH emission arises from the surrounding sky background, as $\mathrm{PAH}$ emission is common in the Magellanic Cloud ISM. 


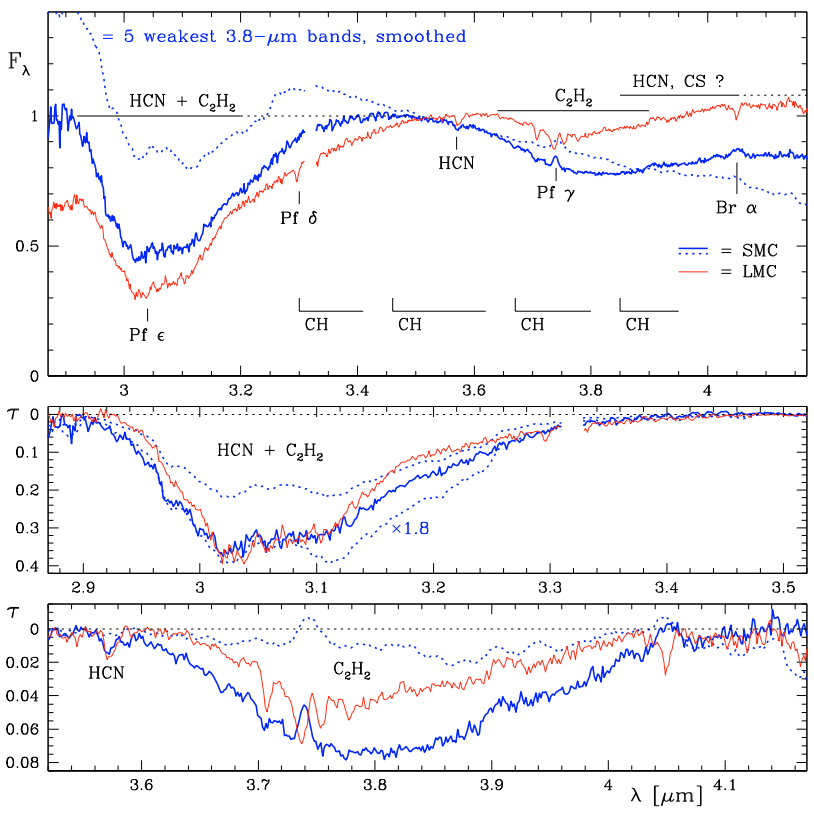

Fig. 9. Average spectrum of the IR carbon stars from Table 1, excluding the "LE" objects in NGC 419 which would not have been selected on their IR properties alone (they are bluer and less dusty). A comparison is made with the LMC (van Loon et al. 2006). Also shown is a smoothed average of the five SMC stars with the weakest 3.8- $\mu \mathrm{m}$ bands (dotted). The bottom two panels show the spectra in close-up, expressed in optical depth with respect to a straight line connecting the spectral points at 2.87 and 3.52 , and 3.52 and $4.17 \mu \mathrm{m}$, respectively.

The O-rich stars are rather unremarkable except for the luminous object PMMR 52, of which a Spitzer spectrum was taken by Lagadec et al. (2007).

The most intriguing serendipitously observed object is MSX SMC 79 B. The signal in the spectrum is weak (Fig. 8), but it clearly displays an extremely red continuum, probably from dust emission, and possibly water ice absorption around $3 \mu \mathrm{m}$ and/or PAH emission around $3.28 \mu \mathrm{m}$. Near to YSO candidate MSX SMC 79 itself, this object too may be an embedded YSO.

\section{Analysis}

\subsection{Carbon chemistry}

A mean of the spectra (after normalising to unity at $3.5 \mu \mathrm{m}$ ) was constructed, of all carbon stars from Table 1 except the NGC 419LE sources, and compared to the equivalent spectrum for the LMC sources from van Loon et al. (2006) to which we also refer the reader for an introduction to the most prominent spectral features (Fig. 9). The hydrogen lines appear weakly in emission in the SMC spectrum and in absorption in the LMC spectrum because of the different way of correcting for the telluric standard star spectrum; these lines should be ignored in either spectrum. There are several noteworthy differences between the SMC and LMC spectra.

Firstly, the LMC spectrum is much redder, as a result of dust extinction and emission. The veiling by the dust emission around $3.6-4 \mu \mathrm{m}$ may be the cause for a weaker $3.8-\mu \mathrm{m}$ band in the LMC compared to the SMC, whilst the $3.1-\mu \mathrm{m}$ band appears approximately equally strong (see also Ohnaka et al. 2007).

Secondly, the $3.8-\mu \mathrm{m}$ acetylene $\left(\mathrm{C}_{2} \mathrm{H}_{2}\right)$ band in the SMC spectrum peaks slightly longward of $3.80 \mu \mathrm{m}$ whereas in the LMC spectrum it peaks slightly shortward of $3.80 \mu \mathrm{m}$. This may be related to the difference in the $3.70-\mu \mathrm{m}$ feature, which is very weak in the SMC compared to the LMC. This is one of a few sharp features attributed to acetylene at low pressure (colder gas at higher elevation - van Loon et al. 2006), and may thus help unravel the connection between the dust formation and the underlying molecular atmosphere. In the LMC, the 3.70- $\mu \mathrm{m}$ feature correlates with a "knee" between 3.15-3.20 $\mu$ m (van Loon et al. 2006), which is indeed also weaker in the SMC.

Thirdly, the HCN band at $3.57 \mu \mathrm{m}$ is weaker in the SMC than in the LMC, possibly as a result of a lower nitrogen abundance in the metal-poor SMC. On the other hand, it appears as if the satellite band of $\mathrm{HCN}$ at $3.58-3.59 \mu \mathrm{m}$ is relatively strong in the SMC carbon stars.

The average of the five SMC carbon stars with the weakest 3.8- $\mu \mathrm{m}$ bands is shown for comparison (Fig. 9, dotted). These stars have an even bluer continuum than the SMC sample as a whole, suggesting that dust is largely absent in the absence of the $3.8-\mu \mathrm{m}$ band. Note that some weak absorption is still discernible near $3.9 \mu \mathrm{m}$. The $3.1-\mu \mathrm{m}$ band is also weaker, but the shape is similar to that of the other SMC stars; when scaled by a factor 1.8 (Fig. 9, middle panel) the profiles match up very well except that the stars with weak $3.8-\mu \mathrm{m}$ absorption have a deeper longwavelength side to the $3.1-\mu \mathrm{m}$ band, possibly as the molecular atmosphere is less extended and warmer.

\subsubsection{Molecular band strengths}

The equivalent widths of the 3.1-, 3.57-, 3.70- and 3.8- $\mu \mathrm{m}$ bands were measured following their definitions by van Loon et al. (2006) (Table 5). This was done for all the objects in Tables 1-4, not just the carbon stars. The relations between the various band strengths are explored in Fig. 10. Clearly, the carbon stars all have strong 3.1- $\mu \mathrm{m}$ bands which separates them from the noncarbon stars. The only interloper is the noisy spectrum of the YSO candidate MSX SMC 79B. The scatter of the non-carbon stars around zero gives an indication of the typical errorbars in the individual equivalent width measurements.

The 3.8- $\mu \mathrm{m}$ band becomes visible in carbon stars with $W_{3.1} \gtrsim$ $1000 \AA$, and then correlates with 3.1- $\mu \mathrm{m}$ band strength (Fig. 10). Remarkably, this trend is indistinguishable between the SMC and LMC. Equally remarkable, the SMC and LMC objects span the same range in equivalent width, for all bands concerned. This is even true for the 3.57- and 3.70- $\mu \mathrm{m}$ bands, although there are fewer SMC carbon stars with substantial ( $W \gtrsim 4 \AA$ ) $\mathrm{HCN}$ absorption at $3.57 \mu \mathrm{m}$ and acetylene absorption at $3.70 \mu \mathrm{m}$ than in the LMC, resulting in weaker features in the average SMC spectrum of Fig. 9; some have negative values, resulting from inaccuracies in defining the continuum in noisy spectra with weak (or absent) absorption features.

The stars with the strongest $3.57-\mu \mathrm{m} \mathrm{HCN}$ band are also the ones with the strongest $3.1-\mu \mathrm{m}$ band, which has a contribution from HCN (Fig. 10); the same is seen in the LMC. However, the reverse is not true: the most negative values for the $3.57-\mu \mathrm{m}$ band are amongst the strongest 3.1- $\mu \mathrm{m}$ absorbers too (Fig. 10); this is not seen in the LMC. Higher resolution and lower noise levels are required before interpreting the negative values as the $\mathrm{HCN}$ band appearing in emission.

The 3.70- $\mu \mathrm{m}$ acetylene band is stronger in stars with strong 3.1- $\mu \mathrm{m}$ bands, which has a contribution from acetylene (Fig. 10). However, the stars with the strongest $3.1-\mu \mathrm{m}$ bands do not show the sharp 3.70- $\mu \mathrm{m}$ feature. An identical trend is seen in the SMC and LMC. 
Table 5. Equivalent widths (in $\AA$ ) of carbonaceous bands.

\begin{tabular}{|c|c|c|c|c|}
\hline Name & $W_{3.1}$ & $W_{3.57}$ & $W_{3.70}$ & $W_{3.8}$ \\
\hline \multicolumn{5}{|l|}{ IR carbon stars } \\
\hline IRAS 00271-7120 & 1407 & 2.50 & 7.97 & 607 \\
\hline GM 780 & 1253 & 7.73 & 1.82 & 327 \\
\hline IRAS 00388-7401 & 1358 & 0.68 & 8.87 & 499 \\
\hline IRAS $00393-7326$ & 1819 & 4.94 & -2.03 & 971 \\
\hline MSX SMC 54 & 1481 & 1.64 & 8.53 & 558 \\
\hline MSX SMC 44 & 1512 & 4.91 & 3.48 & 611 \\
\hline MSX SMC 105 & 1506 & 8.95 & 3.85 & 778 \\
\hline MSX SMC 36 & 1556 & -3.24 & 0.93 & 518 \\
\hline IRAS 00448-7332 & 1113 & 1.44 & 1.38 & 640 \\
\hline MSX SMC 33 & 448 & -0.73 & 0.37 & 127 \\
\hline \multirow[t]{2}{*}{ IRAS 00454-7257 } & 1216 & 0.69 & 4.62 & 309 \\
\hline & 1230 & 0.68 & -0.89 & 376 \\
\hline IRAS 00468-7341 & 1110 & 2.85 & 7.75 & 350 \\
\hline MSX SMC 66 & 1189 & 0.64 & -1.68 & 234 \\
\hline RAW 631 & 668 & 2.35 & 0.86 & 51 \\
\hline MSX SMC 163 & 1499 & 6.45 & 4.54 & 430 \\
\hline ISO-MCMS J005149.4-731315 & 1325 & 0.21 & -0.32 & 372 \\
\hline MSX SMC 125 & 564 & 2.64 & 0.60 & -16 \\
\hline GM 106 & 966 & -1.44 & 0.90 & 95 \\
\hline MSX SMC 159 & 1203 & 1.85 & 2.68 & 413 \\
\hline LEGC 105 & 1435 & 1.65 & -1.17 & 371 \\
\hline ISO-MCMS J005450.7-730607 & 1148 & 3.52 & 2.65 & 154 \\
\hline ISO-MCMS J005454.1-730318 & 780 & 3.66 & 0.03 & 172 \\
\hline MSX SMC 209 & 1365 & -2.99 & 1.13 & 401 \\
\hline IRAS 00554-7351 & 1185 & 3.53 & 6.68 & 388 \\
\hline ISO-MCMS J005720.6-731245 & 1073 & 0.90 & -0.68 & 116 \\
\hline IRAS 00557-7309 & 1328 & 0.97 & 1.52 & 457 \\
\hline MSX SMC 93 & 1759 & -2.76 & 1.55 & 325 \\
\hline NGC 419 LE 16 & 1023 & -1.22 & 2.91 & 116 \\
\hline NGC 419 IR1 & 1310 & 0.22 & -0.84 & 172 \\
\hline NGC 419 IR2 & 1618 & -4.42 & 6.58 & 553 \\
\hline NGC 419 LE 35 & 1680 & 5.51 & 1.76 & 164 \\
\hline NGC 419 LE 27 & 1242 & -0.43 & 0.95 & 121 \\
\hline NGC 419 LE 18 & 1241 & 1.92 & 1.17 & 10 \\
\hline GM 522 & 996 & 2.73 & -2.06 & 84 \\
\hline IRAS 01091-7320 & 1179 & 3.66 & 6.83 & 450 \\
\hline IRAS 01210-7125 & 1722 & -2.61 & -3.09 & 607 \\
\hline \multicolumn{5}{|l|}{ Oxygen-rich AGB stars and RSGs } \\
\hline HV 1375 & -203 & 1.35 & 1.24 & 164 \\
\hline MSX SMC 18 & -137 & 0.79 & 3.01 & -199 \\
\hline BFM 1 & 55 & 0.54 & -0.38 & 41 \\
\hline HV 11262 & -38 & 1.10 & 3.95 & 135 \\
\hline PMMR 34 & 30 & 0.95 & 3.64 & 82 \\
\hline IRAS 00483-7347 & -82 & 0.85 & 2.11 & -261 \\
\hline GM 103 & 33 & 1.47 & 1.12 & -230 \\
\hline PMMR 41 & 31 & -1.72 & 0.24 & 257 \\
\hline BMB-B 75 & -105 & 1.18 & 0.20 & -190 \\
\hline HV 1652 & -549 & 3.58 & -0.05 & 63 \\
\hline HV 11417 & 18 & 1.67 & 3.70 & -449 \\
\hline IRAS 01066-7332 & -179 & -3.00 & -0.58 & -517 \\
\hline HV 12956 & -81 & -1.02 & 2.67 & -185 \\
\hline HV 2084 & 8 & -1.33 & -4.34 & 423 \\
\hline \multicolumn{5}{|l|}{$R C r B$-type stars } \\
\hline MSX SMC 14 & 211 & -3.11 & -0.97 & -24 \\
\hline MSX SMC 155 & -54 & 0.60 & 3.94 & -25 \\
\hline \multicolumn{5}{|l|}{ Post-AGB objects } \\
\hline MSX SMC 29 & -338 & 3.64 & 0.61 & 7 \\
\hline IRAS 00350-7436 & -50 & -0.89 & 0.95 & -12 \\
\hline \multicolumn{5}{|l|}{ Candidate YSOs } \\
\hline MSX SMC 79 & 5 & 10.40 & 0.42 & -110 \\
\hline IRAS 01039-7305 & -173 & -1.48 & -0.33 & -35 \\
\hline IRAS 01042-7215 & 438 & 0.66 & -1.00 & -120 \\
\hline \multicolumn{5}{|l|}{ Serendipitous objects } \\
\hline 2MASS J00472001-7240350 & 144 & 0.95 & 2.55 & 112 \\
\hline 2MASS J00473917-7304115 & 1018 & 4.36 & 1.13 & 95 \\
\hline MSX SMC 79 B & 1546 & 18.72 & -24.48 & 9 \\
\hline RAW 616 & -208 & 3.41 & -1.48 & -77 \\
\hline RAW 639 & 667 & 1.40 & -3.68 & 90 \\
\hline 2MASS J00513146-7310513 & 45 & -5.54 & 7.17 & 164 \\
\hline PMMR 52 & 64 & 2.01 & 2.70 & 207 \\
\hline 2MASS J00544039-7313407 & 1 & -4.48 & 1.37 & 46 \\
\hline PMMR 79? & -293 & 1.15 & 3.76 & 183 \\
\hline 2MASS J00565642-7335301 & 1747 & 2.20 & 12.34 & 560 \\
\hline RAW 1030 & -288 & -3.05 & 6.27 & 84 \\
\hline PMMR 116 & -244 & -1.72 & 3.20 & 23 \\
\hline
\end{tabular}

The SMC and LMC stars differ in the relation between the sharp 3.70- and broad 3.8- $\mu \mathrm{m}$ acetylene bands (Fig. 10): the $3.70-\mu \mathrm{m}$ band strength peaks at a larger $3.8-\mu \mathrm{m}$ band strength in the SMC. Veiling by dust emission would affect both bands about equally, so there must be a more delicate difference between the warmer lower layers and the colder elevated layers in the molecular atmosphere. The SMC stars seem to have more trouble in getting the acetylene to higher elevations than the LMC stars. Alternatively, the $3.70-\mu \mathrm{m}$ band may form throughout the dust envelope, in which case the difference is merely the result of stronger veiling of the $3.8-\mu \mathrm{m}$ band in the dustier LMC stars compared to the less dusty SMC stars. IRAS 00393-7326 has the strongest 3.1- and 3.8- $\mu \mathrm{m}$ bands and is not particularly red $\left(K_{\mathrm{s}}-L_{\mathrm{NB}}^{\prime}=2.6 \mathrm{mag}\right)$, but it does not show any $3.70-\mu \mathrm{m}$ absorption. It does show a clear $3.57-\mu \mathrm{m} \mathrm{HCN}$ absorption feature.

The $3.8-\mu \mathrm{m}$ band may have a contribution from other molecules than acetylene, in particular molecules with $\mathrm{C}-\mathrm{H}$ bonds and possibly carbon sulfide (CS) around $3.9 \mu \mathrm{m}$. To investigate this, the $3.8-\mu \mathrm{m}$ band is split up into a $3.75-\mu \mathrm{m}$ band running from 3.60 to $3.90 \mu \mathrm{m}$, and a 3.9- $\mu \mathrm{m}$ band running from 3.85 to $4.03 \mu \mathrm{m}$ (note the slight overlap). The continuum is determined in both cases by a straight line in the $F_{\lambda}$ spectrum between the medium flux density at 3.50-3.60 $\mu \mathrm{m}$ and that at $4.03-4.10 \mu \mathrm{m}$. We find that in fact both bands correlate extremely well (Fig. 11), suggesting that the contribution from other molecules, in particular CS, may be small. The SMC and LMC stars show the same behaviour, apart from a slight offset at weak bandstrengths - this could be due to a contribution to the $3.75-\mu \mathrm{m}$ band from the $3.70-\mu \mathrm{m}$ absorption components, which peak at lower 3.8- $\mu \mathrm{m}$ bandstrength in the LMC than in the SMC as discussed above. The LMC outlier is BMB-R 46 (SHV 0521050-690415; Matsuura et al. 2005), whose spectrum is extremely noisy around $4 \mu \mathrm{m}$.

\subsubsection{Molecules and dust}

The dust envelope causes extinction below $4 \mu \mathrm{m}$ and possibly emission from $3 \mu \mathrm{m}$ onwards. The $K-L$ colour can be used as a proxy for the optical depth of the dust envelope: Fig. 12 shows a good correlation between the $K_{\mathrm{S}}-L_{\mathrm{NB}}^{\prime}$ colour and the [6.4]-[9.3] colour defined in Sloan et al. (2006) and Zijlstra et al. (2006) for the carbon stars with published Spitzer IRS spectra. These authors show that the [6.4]-[9.3] colour is an accurate measure of the dust optical depth. By inference, the $K-L$ colour is hereby proven to be a good measure of the dust optical depth too. Although a few SMC carbon stars are very red, the LMC carbon stars are generally significantly redder, despite similar molecular band strength (Fig. 13). This suggests that although SMC and LMC stars are similarly proficient in producing molecules, the metal-poor SMC stars are less able to form dust from it.

The dustiest LMC stars clearly have relatively weak $3.8-\mu \mathrm{m}$ bands (Fig. 13), which is evidence of the veiling effect that the dust continuum emission has on the equivalent width of the absorption band. This shows that most of the 3.8- $\mu \mathrm{m}$ band is formed in the warmer layers below the dust envelope. Emission from the molecular bands themselves in strongly pulsating stars can add to the veiling (Hron et al. 1998; Ohnaka et al. 2007). The same may explain the $3.1-\mu \mathrm{m}$ band, which is only moderately strong in the reddest carbon stars as opposed to the large spread in band strengths observed amongst bluer carbon stars (Fig. 13) the latter is also seen in the Milky Way (e.g., Le Bertre et al. 2005). 

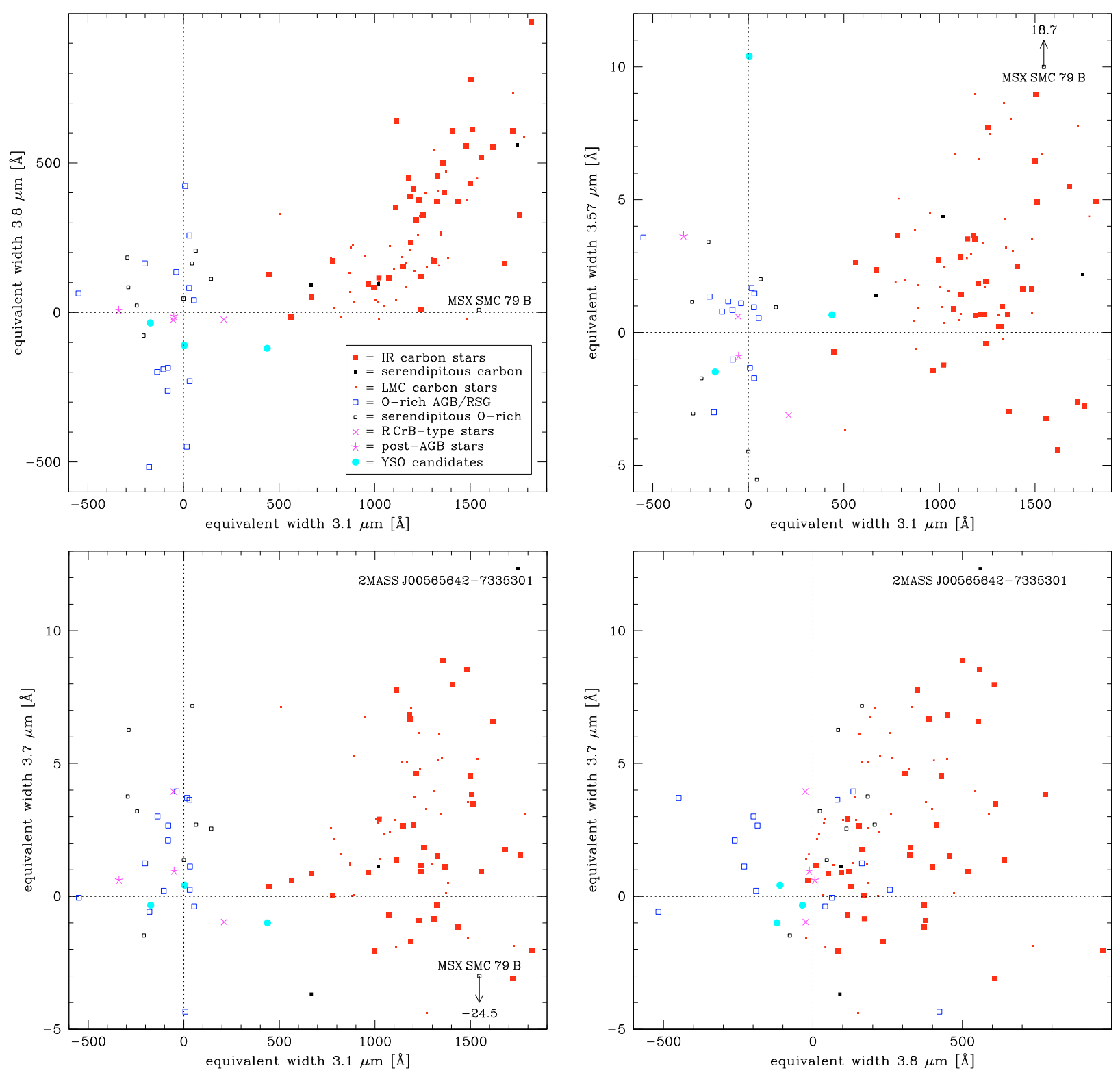

Fig. 10. Equivalent widths (in $\AA$ ) of the 3.1-, 3.57-, 3.70- and 3.8- $\mu$ m molecular bands. Carbon stars in the SMC and LMC show identical trends and cover identical ranges, apart from a slight difference in the $W_{3.7}$ versus $W_{3.8}$ diagram. The other objects mostly scatter around zero, as expected.

On the other hand, the 3.70- $\mu \mathrm{m}$ band clearly reaches maximum strength in the dustier objects, both in the SMC and LMC, which suggests an intimate relation between the dust and the colder acetylene gas at high elevation. This reinforces our previous observation (Sect. 4.1) that dust is not apparent in stars that show little acetylene, and suggests acetylene as a prime building block for carbonaceous grain formation. The star with the strongest $3.70-\mu \mathrm{m}$ band is 2MASS J00565642-7335301; it is not red at all, but its (serendipitous) spectrum is rather noisy and the 3.70- $\mu \mathrm{m}$ band may have been overestimated. Veiling might also be the reason why the stars with the strongest $3.57-\mu \mathrm{m}$ band are the least dusty (Fig. 13). This suggests that the HCN responsible for the $3.57-\mu \mathrm{m}$ feature is present predominantly close to the stellar surface.

\subsubsection{Evolutionary sequence in NGC 419}

NGC 419 offers known initial masses and metallicity. We can place the cluster members in an evolutionary sequence, as on the thermal-pulsing AGB differences in time are more important than differences in initial mass. Assuming that the thickest dust envelopes are formed in the final stages of AGB evolution, the $K-L$ colour can serve as a proxy for evolutionary stage ("time").

Despite the small number of objects, the trends in the band strengths with time confirm our previous analysis (Fig. 14). The $3.57-\mu \mathrm{m}$ band is only discernible in one of the least dusty stars, and then disappears. The 3.1- $\mu \mathrm{m}$ band shows little correlation; this may be due to the fact that the band is saturated and both $\mathrm{HCN}$ and acetylene contribute. On the other hand, the 3.8- $\mu \mathrm{m}$ band is strong only in the dustiest star. The 3.70- $\mu \mathrm{m}$ band also becomes stronger as the dust envelope thickens. This suggests that, as the molecular atmosphere thickens (as a result of lower temperature and/or higher $\mathrm{C} / \mathrm{O}$ ratio), more acetylene is brought to higher elevation and more dust is formed. The elevation of the molecular atmosphere is probably caused by the stronger pulsation as the star evolves (and cools).

The two stars, IR1 and IR2, in the similar cluster NGC 1978 in the LMC (van Loon et al. 2005b, 2006) (small symbols in 


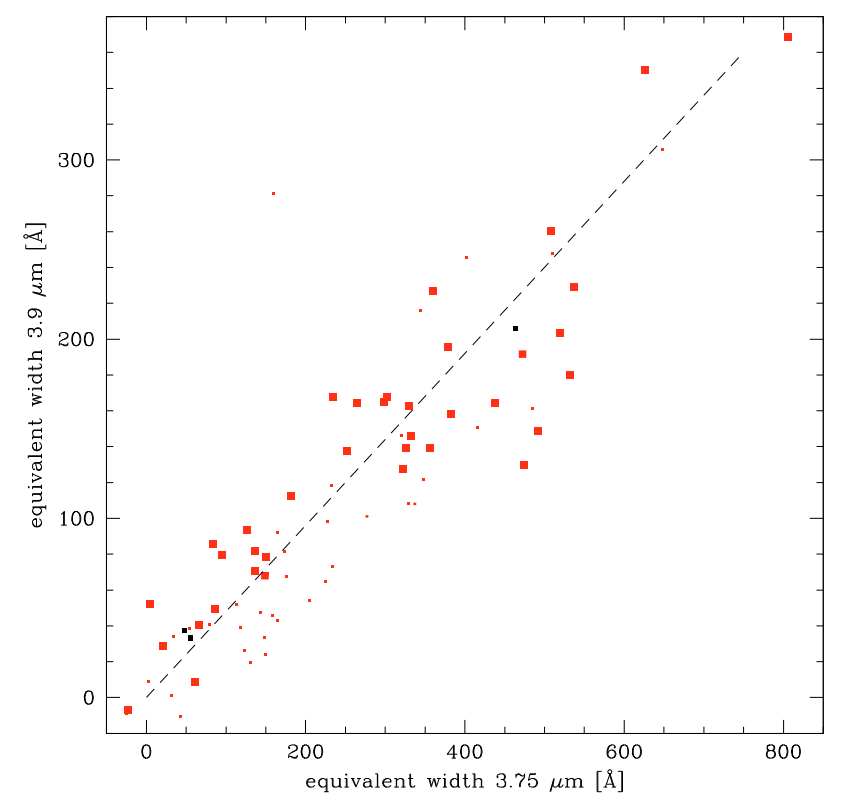

Fig. 11. Equivalent widths (in $\AA$ ) of the 3.75- and 3.9- $\mu$ m molecular bands (symbols are as in Fig. 10). The dashed line is for reference only.

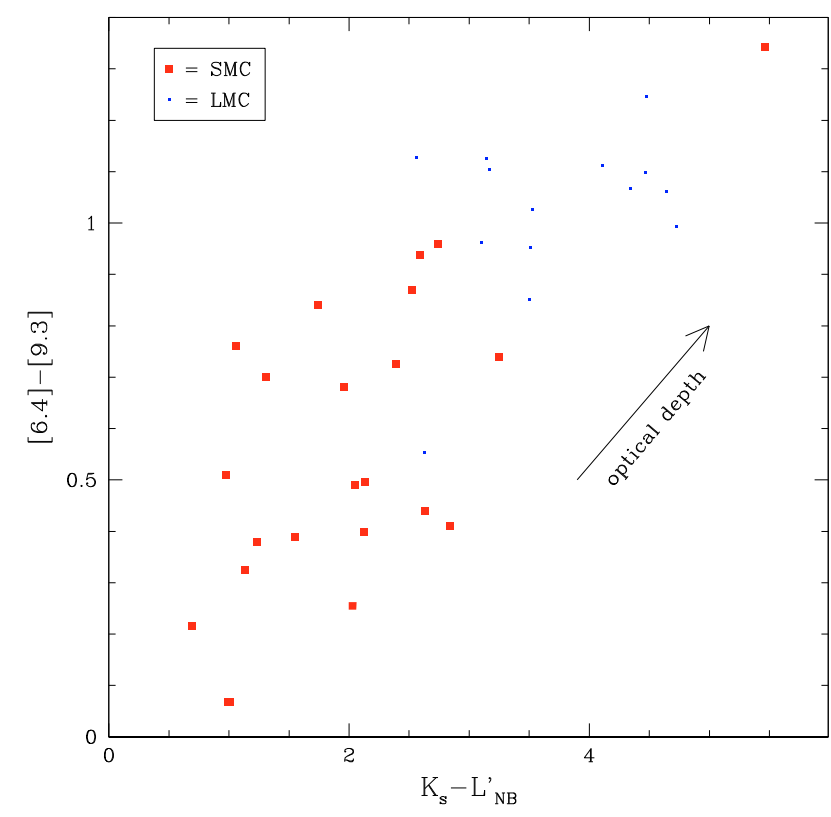

Fig. 12. Correlation for carbon stars, between the $K_{\mathrm{s}}-L_{\mathrm{NB}}^{\prime}$ colour and the [6.4]-[9.3] colour defined for Spitzer IRS spectra by Sloan et al. (2006) and Zijlstra et al. (2006). The latter is an accurate measure of the optical depth.

Fig. 14) confirm exactly the same behaviour as seen in NGC 419. This is not surprising, as de Freitas Pacheco et al. (1998) measured identical metallicities of $[\mathrm{Fe} / \mathrm{H}]=-0.60 \pm 0.21$ for both clusters, although they find that NGC 419, at $1.2 \pm 0.5 \mathrm{Gyr}$, is somewhat younger than NGC 1978, at 3.0 \pm 1.0 Gyr. The associated difference in mass of the AGB stars in these clusters apparently is not large enough to lead to noticeable differences in their molecular atmospheres.

\subsubsection{Polycyclic Aromatic Hydrocarbons}

The post-AGB object MSXSMC 29 shows the best example of the $3.28-\mu \mathrm{m}$ band attributed to emission from PAHs
(Fig. 5), stronger than in the other SMC post-AGB object, IRAS 00350-7436 (Matsuura et al. 2005). Its $K_{\mathrm{s}}-L_{\mathrm{NB}}^{\prime}$ colour is somewhat bluer than the loosely defined sequence of dustenshrouded carbon stars (Fig. 1); it is also bluer than the two $\mathrm{R}$ CrB-type stars in our sample, and brighter in the near-IR than the reddest of these two. The central star of IRAS 00350-7436 has a G- or K-type optical spectrum with strong CN bands, and it is about as luminous as AGB stars get, $L \sim 50000 \mathrm{~K}$ (Whitelock et al. 1989; Groenewegen et al. 2000) - the faint near-IR magnitudes are a result of the relatively large near-IR bolometric corrections for this warm star. This suggests a relatively massive AGB star progenitor, which is intriguing given that it is a carbon-rich object. HBB prevents massive AGB stars, with initial masses in excess of $\sim 4 M_{\odot}$, from becoming a carbon star (Boothroyd et al. 1993). On the other hand, once mass loss has almost completely depleted the stellar mantle, HBB will cease and another thermal pulse could still turn the star into a carbon star (Frost et al. 1998). The latter scenario predicts that the carbon-rich dust shell is enveloped by a much older, cooler oxygen-rich dust shell.

MSX SMC 29 was observed with the Spitzer IRS by Kraemer et al. (2006), who detect several PAH emission bands in the 6-13 $\mu \mathrm{m}$ region, as well as absorption from acetylene and larger hydrocarbons. They interpret the presence of aliphatic bonds as evidence of a mild ionization environment, placing the object to within only a few centuries since its departure from the AGB. Aliphatic bonds are expected to produce additional PAH emission at $3.4 \mu \mathrm{m}$ (Sloan et al. 2007); this is not seen in our $L$-band spectrum (Fig. 5), but the spectrum does show a depression where this feature is expected, suggesting that it might be in absorption. Such an unusual result requires confirmation with higher signal-to-noise data.

R CrB-type stars are hydrogen-poor, and believed to sporadically form carbon-rich dust (Clayton 1996), whilst the heating central stars of post-AGB objects have stopped forming fresh dust. R CrB-type stars may well be the "proto-post-AGB" objects that are just leaving the AGB. There may thus exist an evolutionary sequence from optically bright carbon stars $\rightarrow$ dust-enshrouded carbon stars $\rightarrow$ R CrB-type stars $\rightarrow$ carbonrich post-AGB objects, where the PAHs appear as the central star of the post-AGB object becomes hot enough to destroy the dust grains that were formed not long before during the AGB and/or R CrB phase, and to excite the energetic transitions of the PAHs (cf. Sloan et al. 2007). However, recent measurements of greatly enhanced ${ }^{18} \mathrm{O}$ in $\mathrm{R} \mathrm{CrB}$ stars have been taken as evidence against a very late helium-flash scenario (such as occurring on the white dwarf cooling track), possibly pointing instead at a double-degenerate merger origin (Clayton et al. 2007).

\subsection{Oxygen chemistry}

The cooler, M-type stars show a curvature resulting from molecular absorption, mainly of water $\left(\mathrm{H}_{2} \mathrm{O}\right)$ and silicon monoxide (SiO) (e.g., Tsuji et al. 1997; Yamamura et al. 1999; Decin \& Eriksson 2007). A simple way of quantifying this is obtained by averaging the flux densities at 3 and $4 \mu \mathrm{m}$, and comparing it to the flux density at $3.5 \mu \mathrm{m}$ :

$S_{\text {mol }}=2 F_{3.5} /\left(F_{3}+F_{4}\right)-1$.

A strongly curved spectrum due to abundant molecules yields a positive value for this "molecular index", whilst a spectrum with little molecular absorption yields a negative value. The computed values are listed in Table 6. 

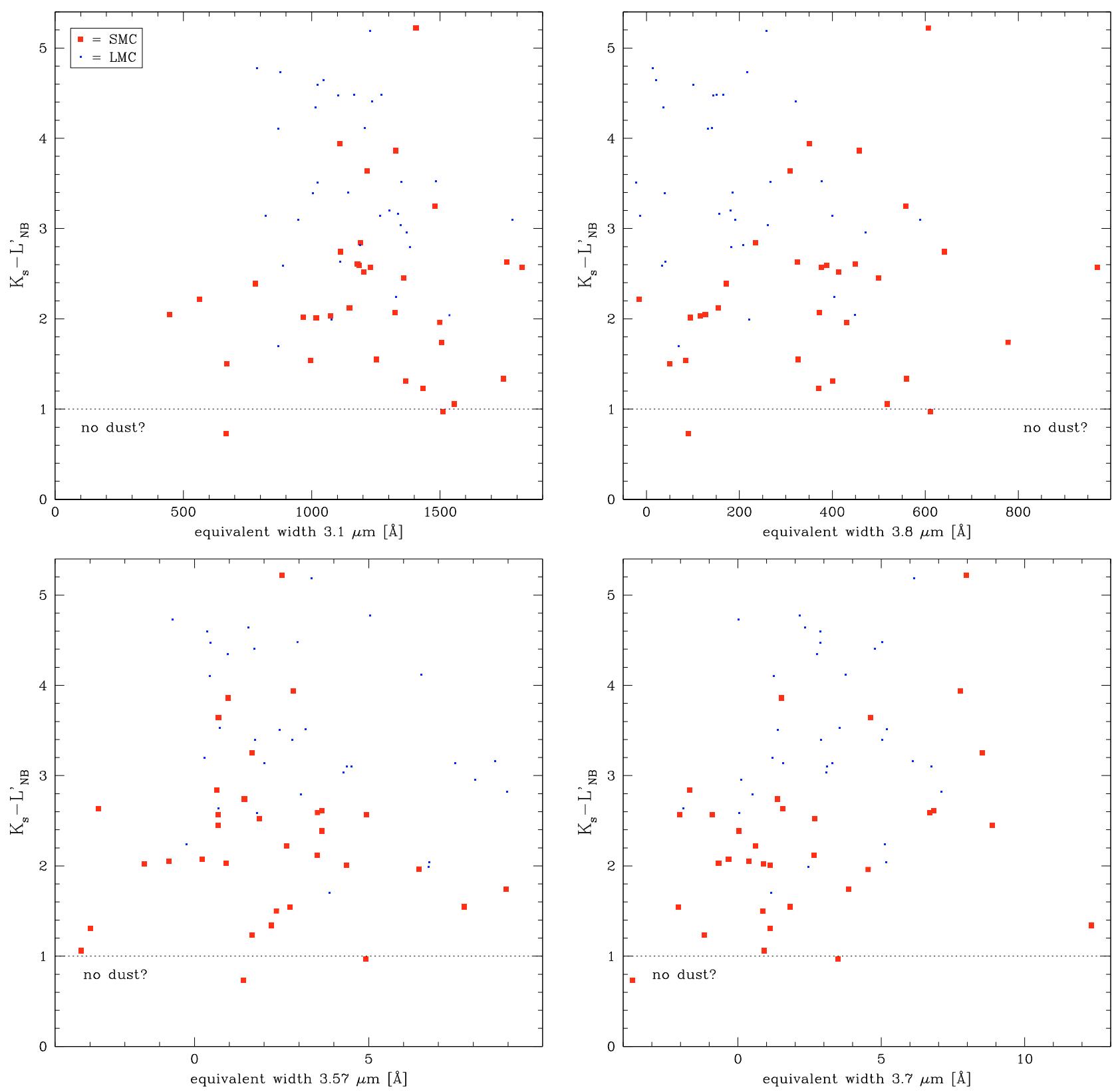

Fig. 13. Dependence of the $K_{\mathrm{s}}-L_{\mathrm{NB}}^{\prime}$ colour, a proxy for the optical depth of the circumstellar dust envelope, and the equivalent widths (in $\AA$ ) of the 3.1-, 3.57-, 3.70- and 3.8- $\mu$ m molecular bands. The differences between carbon stars in the SMC and LMC may reflect a different connection between the gas and the dust. Stars from the NGC 419 cluster are not included (see Fig. 13).

Stars that do not show red $K_{\mathrm{S}}-L_{\mathrm{NB}}^{\prime}$ colours indicative of circumstellar dust tend to have $L$-band spectra that are not affected by the molecular bands, and vice versa (Fig. 15, left). The redder stars in the SMC sample do show the molecular bands. This could be associated with a more advanced evolutionary state of the dustier stars, when they are cooler and possibly stronger pulsation extends the molecular atmosphere. Indeed, all SMC stars with spectral types later than M2 show the molecular signature in their spectra (see Table 6). The S-type star in our sample, BFM 1 does not show the molecular bands, possibly because its $\mathrm{C} / \mathrm{O}$ ratio is close to unity and there is thus little oxygen left after formation of $\mathrm{CO}$ to form water (and $\mathrm{SiO}$ ).

In contrast to some of the SMC stars, the stars in our LMC comparison sample (Matsuura et al. 2005; van Loon et al. 2006) do not show any effect of the molecular bands on their $L$-band spectra (Fig. 15, left). This could be a selection bias.
However, the M9-type AGB star IRAS 05128-6455 (van Loon et al. 2005a) is very cold and would thus be expected to show strong molecular bands. Possibly the dust continuum emission veils these bands; dust emission is certainly very prominent in the RSG OH/IR star IRAS 05280-6910 (NGC 1984 IR1; van Loon et al. 2005b). It is not obvious why SMC stars with similar $K_{\mathrm{s}}-L_{\mathrm{NB}}^{\prime}$ colours should show the spectral curvature associated with strong molecular bands.

Series of equally-spaced lines of hydroxyl $(\mathrm{OH})$ cover parts of the $L$-band. These lines are narrow and weak, and hard to see in our spectra. To enhance our ability to detect $\mathrm{OH}$, we shift-average the spectra such that six of the stronger, isolated OH lines coincide: $\mathrm{OH} 1-0 \mathrm{P} 13-16$ and $\mathrm{OH} 2-1 \mathrm{P} 14$ and P15 at wavelengths of $3.345,3.400,3.455,3.515,3.570$ and $3.640 \mu \mathrm{m}$, respectively (Wallace \& Hinkle 2002). As a result, $\mathrm{OH}$ is seen in many stars in our sample, with depths ranging between 0 


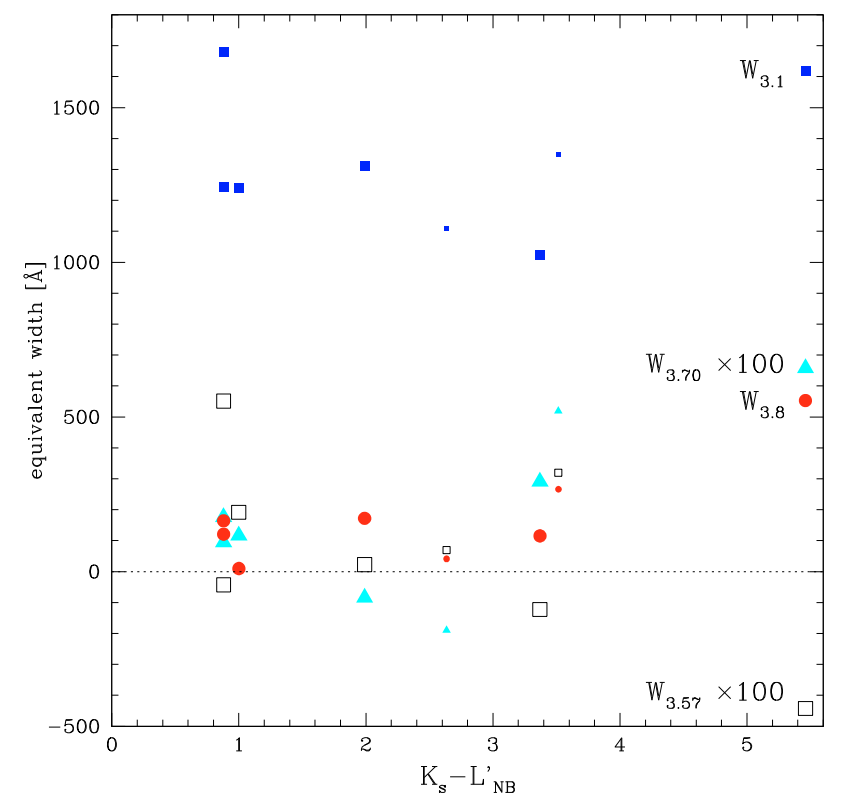

Fig. 14. Equivalent widths (in $\AA$ ) of the 3.1-, 3.57-, 3.70- and 3.8- $\mu \mathrm{m}$ molecular bands as a function of the $K_{\mathrm{S}}-L_{\mathrm{NB}}^{\prime}$ colour, in the populous intermediate-age cluster NGC 419, and (small symbols) in the similar cluster NGC 1978 in the LMC (van Loon et al. 2005b, 2006). The thickest circumstellar dust shells show stronger acetylene absorption, both at 3.8- and $3.70 \mu \mathrm{m}$.

and $\sim 3 \%$ of the local (pseudo-)continuum (Fig. 16). In this respect, there is no difference between the O-rich AGB stars and the RSGs, suggesting that luminosity does not play an important rôle in determining the $\mathrm{OH}$ abundance. This confirms the findings by Wallace \& Hinkle (2002) based on Galactic giants and supergiants. The S-type star, BFM 1 shows no sign of $\mathrm{OH}$ in its spectrum.

The O-rich AGB stars and RSGs in the LMC show a similar range in $\mathrm{OH}$ strength (Fig. 16, dashed), from absent in IRAS 5128-6455 and IRAS 05280-6910 - which have the reddest continua of the O-rich stars in the LMC sample - to a depth of $\sim 5 \%$ in the M4-type RSG HV 2532. The equivalent width of the average of these $\mathrm{OH}$ lines diminishes as the stars become redder (Fig. 15, middle), as expected if the dust continuum veils the $\mathrm{OH}$ lines. The less dusty stars in the LMC show on average stronger $\mathrm{OH}$ absorption than SMC stars, possibly because of the higher oxygen abundance or smaller fraction of oxygen locked up into $\mathrm{CO}$ (or both).

The first overtone rotational-vibrational band of $\mathrm{SiO}$ is seen in some stars. The entire band may be in emission, and is then relatively easy to detect in low-resolution spectra, e.g., HV 2084 (Fig. 6). In a close-up view of this spectral region, the band heads can sometimes be seen in absorption, but this is quite difficult at our signal-to-noise level and low spectral resolution. Examples are 2MASS J00513146-7310513 and PMMR 116, and the absorption components might also be seen superimposed on the emission in HV 1375 (Fig. 17). We list possible SiO detections in Table 6.

Matsuura et al. (2005) show with medium-resolution spectra that luminous AGB stars and RSGs in the LMC show hardly any trace of $\mathrm{SiO}$. Veiling by dust and/or chromospheric continuum emission, and/or band reversal due to pulsational shocks may have made the $\mathrm{SiO}$ bands appear weaker in their small sample (an example of filling-in of the absorption by diffuse emission is given by Sloan \& Price (1998) for $\alpha$ Herculis).
Table 6. Oxygenous band strengths in AGB stars and RSGs. The $\mathrm{SiO}$ band may appear in absorption (A) or emission (E).

\begin{tabular}{lrrll}
\hline \hline Name & $S_{\mathrm{mol}}$ & $W_{\mathrm{OH}}$ & $\mathrm{SiO}$ & $\mathrm{SpT}$ \\
\hline SMC & & & & \\
HV 1375 & 0.03 & 1.91 & $\mathrm{E}+\mathrm{A}$ & $\mathrm{M} 5$ \\
MSX SMC 18 & 0.12 & 0.44 & $\mathrm{~A} ?$ & \\
BFM 1 & -0.06 & 0.36 & & $\mathrm{~S}$ \\
HV 11262 & -0.04 & 3.66 & & $\mathrm{M} 0.5$ \\
PMMR 34 & -0.10 & 4.32 & $\mathrm{E} ?$ & $\mathrm{M} 0.5$ \\
IRAS 00483-7347 & 0.22 & 2.77 & $\mathrm{~A} ?$ & $\mathrm{M} 8$ \\
GM 103 & 0.21 & 0.89 & $\mathrm{~A} ?$ & $\mathrm{M} 4$ \\
PMMR 41 & -0.15 & 3.67 & $\mathrm{E}$ & $\mathrm{M} 0 \mathrm{Iab}$ \\
BMB-B 75 & 0.08 & 0.32 & $\mathrm{~A}$ & $\mathrm{M}$ \\
HV 1652 & -0.09 & 1.40 & $\mathrm{E}$ & $\mathrm{M} 0.5$ \\
HV 11417 & 0.16 & 2.08 & $\mathrm{E} ?$ & $\mathrm{M} 5 \mathrm{e}$ \\
IRAS 01066-7332 & 0.30 & -0.90 & & $\mathrm{M} 8$ \\
HV 12956 & 0.15 & 1.41 & & $\mathrm{M} 5$ \\
HV 2084 & -0.10 & -0.53 & $\mathrm{E}$ & $\mathrm{M} 2 \mathrm{Ia}$ \\
2MASS J00472001-7240350 & -0.13 & 2.69 & $?$ & \\
2MASS J00513146-7310513 & -0.14 & 0.65 & $\mathrm{~A}$ & \\
PMMR 52 & -0.14 & 4.09 & $\mathrm{E}$ & $\mathrm{K} 5$ \\
2MASS J00544039-7313407 & -0.05 & 5.37 & & \\
PMMR 79 ? & 0.14 & 3.30 & $\mathrm{E} ?$ & K5 \\
PMMR 116 & -0.02 & 1.84 & $\mathrm{~A}$ & $\mathrm{~K} 6.5$ \\
LMC & & & & \\
IRAS 05280-6910 & -0.04 & -2.10 & $\mathrm{E}+\mathrm{A}$ & $\mathrm{OH} / \mathrm{IR}$ \\
NGC 1984 IR2 & -0.06 & 3.70 & $?$ & \\
MSX LMC 50 B & -0.09 & 3.66 & $\mathrm{~A}$ & \\
HV 11977 & -0.10 & 2.86 & $\mathrm{~A}$ & $\mathrm{M}$ \\
HV 2532 & 0.00 & 4.00 & $\mathrm{~A}$ & $\mathrm{M} 4 \mathrm{I}$ \\
MSX LMC 1780 B & -0.13 & 4.46 & & \\
IRAS 05128-6455 & -0.16 & -0.29 & & M9 \\
IRAS 05148-6730 & -0.19 & 1.20 & & M2.5 I \\
\hline
\end{tabular}

Indeed, these LMC stars resemble luminous, strongly pulsating stars in the Solar Neighbourhood (Rinsland \& Wing 1982). Our larger compilation of LMC spectra do include some examples of SiO bands, e.g., HV 2532 (Fig. 17). Targets show the band more often in emission, whilst serendipitous spectra show it more often in absorption, likely because the latter tend to have lower luminosity, weaker pulsation and less continuum veiling. Still, Galactic stars regularly show depths in excess of $10 \%$ of the continuum, whereas few, if any, of the SMC or LMC stars show the bands at such level. We thus confirm the tentative conclusion reached by Matsuura et al. (2005), and the firmer conclusion on the basis of the $\mathrm{SiO}$ fundamental band at $8 \mu \mathrm{m}$ in Spitzer spectra by Sloan et al. (2008), that $\mathrm{SiO}$ is weaker at lower metallicity. The reason is likely the lower silicon abundance.

HV 1652 is peculiar. It shows very strong absorption around $2.9 \mu \mathrm{m}$ (Fig. 6), which may be attributed to the $2.7-\mu \mathrm{m}$ water vapour band (Merrill \& Stein 1976). The depression between 3.4 and $3.7 \mu \mathrm{m}$ may be due to $\mathrm{OH}$, or $\mathrm{TiO}$ (Schwenke 1998). There is also a hint of narrow $\mathrm{SiO}$ lines appearing in emission (Fig. 17). The spectral type of this RSG is only M0.5, and its $K_{\mathrm{S}}-L_{\mathrm{NB}}^{\prime}$ is not red at all. It seems to have an extended molecular atmosphere but not to produce much dust. This might be due to a lack of nucleation seeds which rely on secondary metallic elements such as titanium, but if the $3.4-3.7 \mu \mathrm{m}$ depression is caused by $\mathrm{TiO}$ this would not appear to be the case.

\section{Discussion}

We first discuss the formation of molecules and dust in carbonand oxygen-rich evolved stars in the Magellanic Clouds, before discussing their impact on the ISM. 

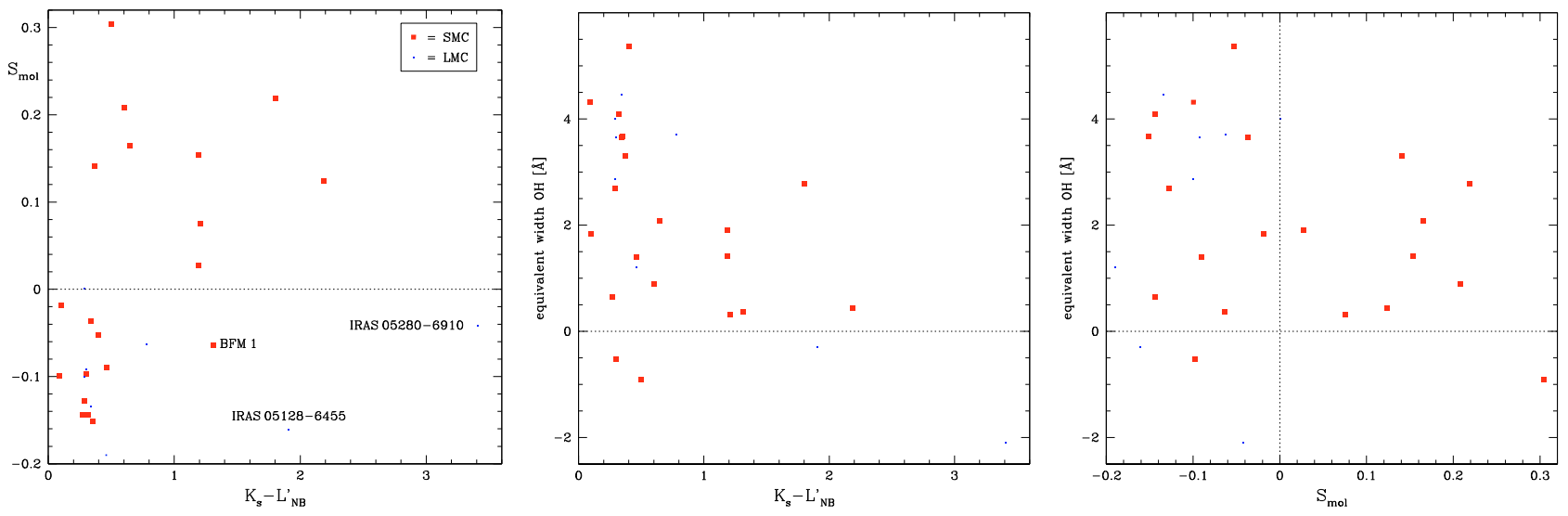

Fig. 15. Molecular index and equivalent width of the $\mathrm{OH}$ lines for O-rich AGB stars and red supergiants in the SMC and LMC.

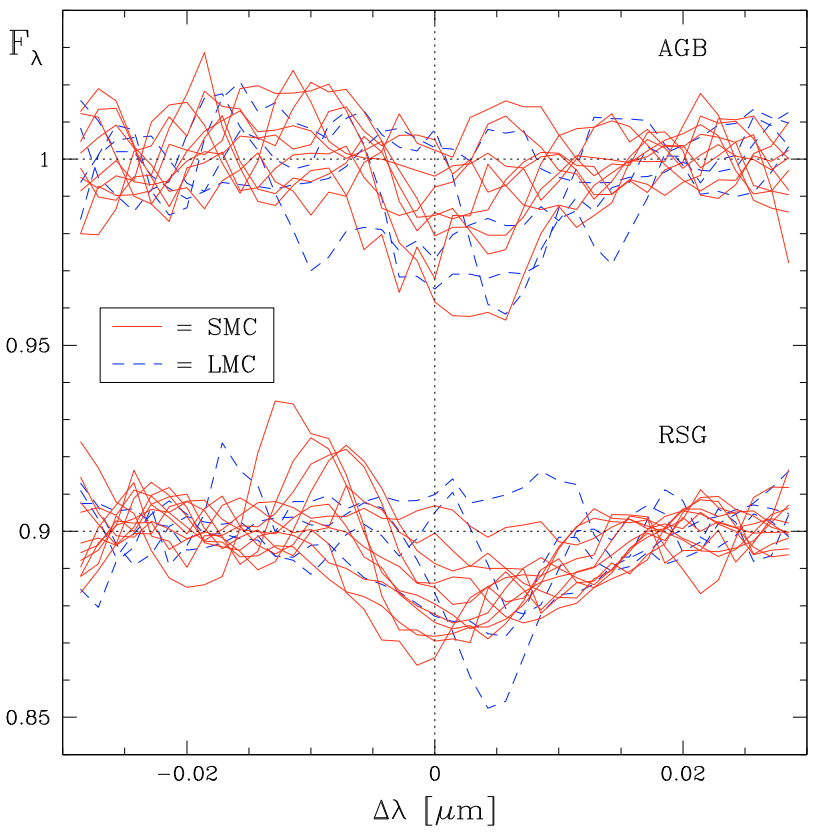

Fig. 16. Averages of six $\mathrm{OH}$ lines (see text), for the O-rich AGB stars and (offset) for the red supergiants. Both groups of stars show a similar range in $\mathrm{OH}$ absorption. This suggests the presence of $\mathrm{OH}$ absorption is not related to luminosity.

\subsection{Molecules and dust in carbon stars}

In the Magellanic Clouds, AGB stars descending from stars that were born 200 Myr to a few Gyr ago become carbon stars (Marigo et al. 1996) before they start producing any significant amounts of dust (van Loon et al. 1999b). Near-IR colours are redder, and veiling of the molecular absorption bands is stronger, in LMC carbon stars compared to SMC carbon stars. This implies that LMC carbon stars are dustier. The molecular bands are equally strong in the SMC though, which suggests that the connection between the molecular atmosphere and the dust envelope is not immediate.

Some SMC carbon stars show as strong absorption by highelevation acetylene gas as carbon stars in the LMC. It thus appears that dust forms less effectively in the SMC from an otherwise similar molecular resource. The reason for this could be the requirement for nucleation seeds to be present, which are likely to contain titanium and other secondary elements not produced by the AGB star itself (e.g., Ferrarotti \& Gail 2006). If

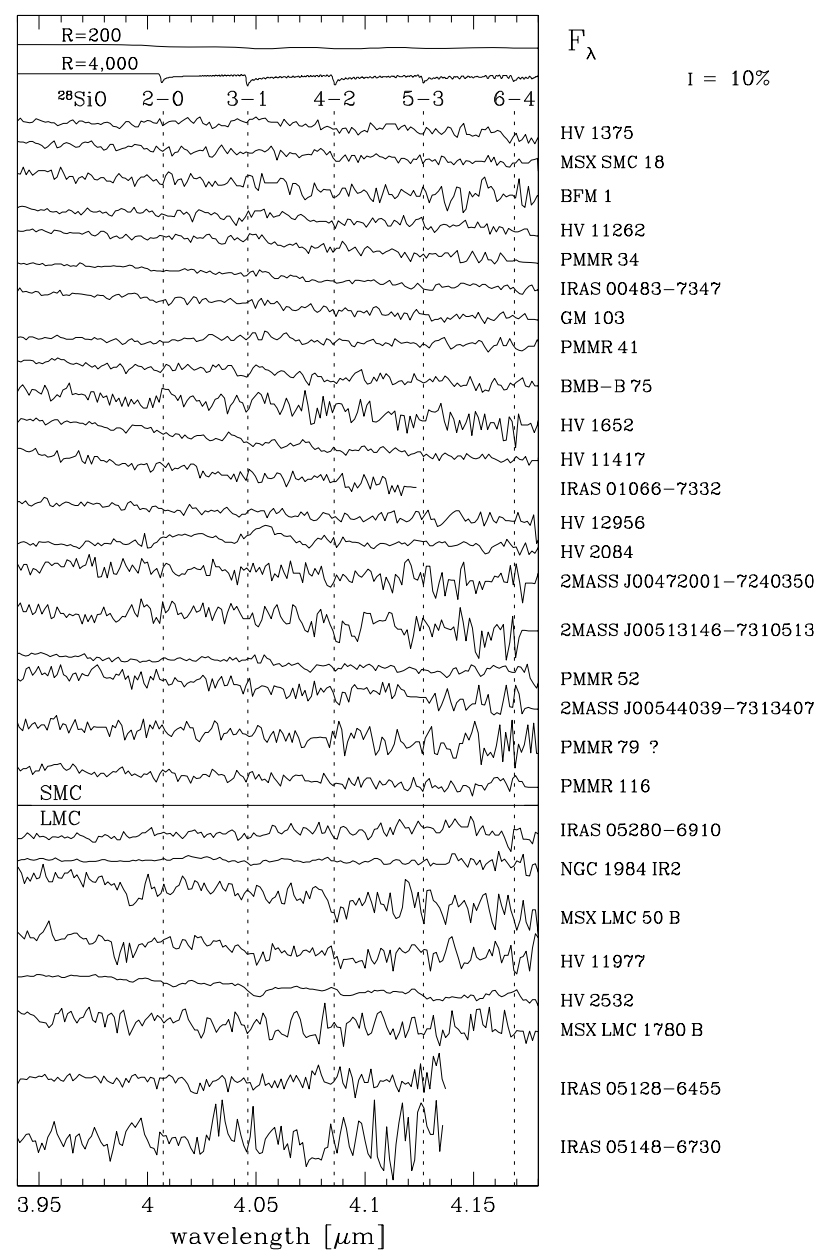

Fig. 17. The SiO first overtone bands of the O-rich AGB stars and red supergiants in the SMC and LMC. For comparison, a synthetic $\mathrm{SiO}$ spectrum for $T=2000 \mathrm{~K}$ is displayed at the top, for two different spectral resolving powers. The vertical dotted lines indicate the positions of the $\mathrm{SiO}$ bandheads.

less molecular material is locked in dust, it is not surprising to find relatively large amounts of remaining molecular gas at high elevation. On the whole, though, the broader absorption bands in the SMC compared to the LMC suggest that more of the molecular gas in SMC carbon stars is found close to the surface of the star (Fig. 9). 
That the molecular atmospheres do not appear to be more massive in the SMC compared to the LMC is in contradiction to the expectations from a simple scaling with the $\mathrm{C} / \mathrm{O}$ ratio (Matsuura et al. 2005). Either the free carbon abundance in the SMC does not exceed that in the LMC, or the column density of molecular gas is limited by other factors such as the temperature and density profile throughout the pulsating atmospheric layers (Mattsson et al. 2008).

Still, it has been suggested that a higher $\mathrm{C} / \mathrm{O}$ ratio in metalpoor carbon stars might lead to more prolific dust production in these stars, which would have important implications for the dust injection rate into the ISM (Matsuura et al. 2005). However, we have just seen that metal-poor carbon stars do not seem to have dustier winds. If they did, then the dust-driven wind scenario (van Loon 2000) predicts that their winds should be faster by $\sqrt{\psi}$, where $\psi$ is the dust-to-gas ratio. For the same total massloss rate, the optical depth of the wind would also increase by the same factor. But the winds of metal-poor carbon stars are not observed to be optically thicker (see also Fig. 12). To reconcile this with a higher dust content, the mass-loss rates of metal-poor carbon stars must then be lower, which would then be difficult to explain. That not more dust is formed in metal-poor carbon stars is also consistent with the very low wind speed measured in Galactic halo carbon stars (Groenewegen et al. 1997; Mauron 2008), and with the lack of dust seen in Planetary Nebulae in the Magellanic Clouds (Stanghellini et al. 2007).

This all favours the notion of similar total mass-loss rates, via less dusty winds, of carbon stars in the metal-poor SMC compared to the metal-richer LMC (van Loon 2000, 2006). The mass-loss rate must be determined by inner boundary conditions such as pulsation - which appears to reach similar strength in both Magellanic Clouds (van Loon 2002; Groenewegen et al. 2007) - whilst the dust production must be determined by secondary elements such as titanium.

\subsection{Molecules and dust in massive AGB stars and in red supergiants}

The most massive AGB stars and the red supergiants are generally oxygen-rich. In the SMC, the coolest of these stars show impressively strong molecular absorption. It is difficult to understand why the molecular abundance should be so high in these metal-poor stars, in particular as it is also seen in some of the RSGs - which are not known to show any surface abundance enhancements of either oxygen or carbon. The bulk of the molecular mass may be closer to the surface of the star than in metalrich stars. This would increase the column density (less dilution) as well as the excitation temperature, both of which would result in a higher rate in the rotational-vibrational transitions, and thus stronger absorption (see Yamamura et al. 1999). It is also possible that the water continuum absorption is optically thick more often in the LMC stars, in which case the curvature is suppressed. This "self-veiling", in combination with that by the dust continuum, could explain the absence of curvature and $\mathrm{OH}$ lines in the most extreme LMC objects (Fig. 15).

The $\mathrm{SiO}, \mathrm{OH}$ and water seen in the $L$-band spectrum arise from regions well within a stellar radius from the stellar photospheric continuum (Tsuji et al. 1997). The best prospects for detecting these molecules further out are through the maser lines of $\mathrm{SiO}$ at $43 \mathrm{GHz}(1-0)$ and $86 \mathrm{GHz}(2-1)$, water at $22 \mathrm{GHz}$, and its dissociation product $\mathrm{OH}$ at $1612 \mathrm{MHz}$; these can be stronger than the thermalised rotational transitions of $\mathrm{CO}$ at $115 \mathrm{GHz}(1-0)$ and $230 \mathrm{GHz}(2-1)$. However, to date no such detections have been made in the SMC, and few in the LMC
(Wood et al. 1992; van Loon et al. 2001; Marshall et al. 2004). This is likely due to the lower molecular abundances as a result of the lower abundances of silicon and oxygen in the Magellanic Clouds, and the lower dust content and hence fainter IR emission and lower pumping rate of the population inversion that drives the stimulated emission in the maser transition.

The fact that $\mathrm{SiO}$ is sometimes seen in emission in the SMC stars implies that pulsation can be strong in these cool stars despite their low metallicity. It is therefore conceivable that similar total amounts of gas are deposited in the region above the stellar photosphere where dust can form. As $\mathrm{SiO}$ forms the basis for the formation of the silicate dust that typically dominates the dust grain population in well-developed winds from AGB stars and RSGs, a lower $\mathrm{SiO}$ molecular abundance in SMC stars is likely to hamper the growth of dust grains in their envelopes. Also, the dust formation is likely to rely on a nucleation seed containing titanium or another secondary element - Nittler et al. (2008) argue that $\mathrm{Al}_{2} \mathrm{O}_{3}$ may condense before $\mathrm{TiO}_{2}$ - which is certain to be less abundant in low-metallicity stars. However, as long as enough dust forms to be coupled dynamically with the gaseous fluid, the gas present in the dust formation zône may be driven away albeit at a slower speed. This is indeed confirmed to happen in OH/IR stars in the Magellanic Clouds compared to the Milky Way (Marshall et al. 2004; van Loon 2006).

\subsection{The rôle of pulsation in driving mass loss}

To quantify the potential of pulsation to drive a wind, van Loon (2002) introduced the mean energy rate involved in the associated luminosity modulation (a result of the $\kappa$-mechanism causing the radial pulsation), taking the $K$-band amplitude as a proxy for a sinusoidal bolometric light modulation (cf. van Loon et al. 2006). This quantity, $\dot{E}_{\text {puls }}$, is mathematically restricted to the range $0-0.5 L_{\star}$, where $0.5 L_{\star}$ would correspond to the star "switching off" altogether at minimum light.

We illustrate in Fig. 18 the energy contained within the pulsation, wind and radiation field using mainly data from the LMC (Whitelock et al. 2003), complemented with data from the South Galactic Cap (Whitelock et al. 1994); very limited data is available for the SMC: carbon stars IRAS 00448-7332, IRAS 00554-7351 and NGC 419 IR2 (Groenewegen et al. 2007) and M-type stars IRAS 00483-7347, HV 11417 and HV 12956 (Whitelock et al. 1989; Elias et al. 1985) - all scaled to consistent distances and dust-driven wind properties (van Loon 2006). There is remarkably little difference in the trends seen amongst these different populations.

The pulsation of AGB stars appears to saturate just short of the maximum attainable storage of photons within the partiallyionized regions of the mantle during expansion (Fig. 18, top), whilst the energy output of RSGs is clearly modulated less strongly. This is reflected in the smaller amplitudes of the lightcurves of RSGs, but in an absolute sense $\dot{E}_{\text {puls }}$ of RSGs rivals that of AGB stars.

The rate at which the wind carries away kinetic energy is $\dot{E}_{\text {wind }}=\dot{M} v^{2} / 2$, where $\dot{M}$ is the mass-loss rate and $v$ is the wind speed. Using the data and scaling relations from van Loon et al. $(1999 \mathrm{~b}, 2005 \mathrm{a})$, the radiation field is found to contain $10^{4}$ to $10^{7}$ times more energy than required to support the wind (Fig. 18, centre), independent of stellar luminosity (cf. Judge \& Stencel 1991). This suggests that something other than radiation determines the mass loss.

The kinetic energy in the wind is tiny ( $\sim 10^{-5}$ times) compared to the energy modulated in the pulsating mantle, and a smooth function of pulsation period, $P$, indistinguishable for 

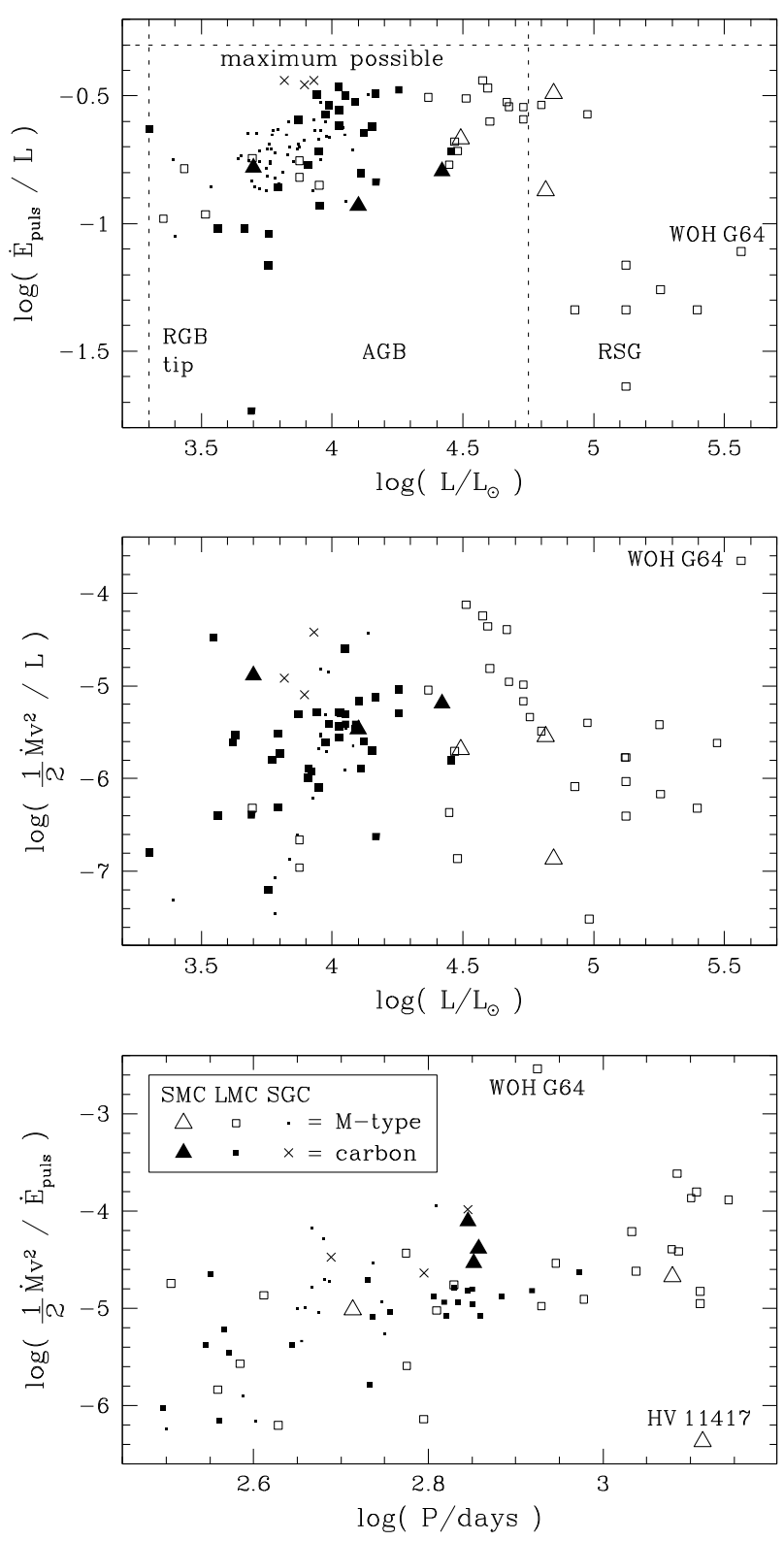

Fig. 18. The mean energy rate involved in pulsational variability as a fraction of luminosity, versus luminosity (top), and the kinetic energy in the ejecta compared to luminosity, versus luminosity (centre) and compared to pulsational energy rate, versus pulsation period (bottom), for carbon- and oxygen-rich AGB stars and RSGs in the SMC (triangles), LMC (squares) and South Galactic Cap (triangles/dots).

M-type and carbon AGB stars (Fig. 18, bottom). WOH G064 is an anomaly; its mass-loss rate is over-estimated due to nonsphericity of the dust envelope (Ohnaka et al. 2008). Perhaps slower pulsations drive a wind more efficiently, if the molecular layers are elevated more if given more time during a pulsation cycle. Another outlier is HV 11417, whose mass-loss rate appears low.

Bowen (1988) found in his pulsation models that the fraction of luminosity that is dissipated by pulsation shocks is of order a per cent. Less than a per cent of this appears to be used to perform work on the expanding layers; hence most of the dissipated energy must leave the system through radiation. Indeed, modulated $\mathrm{H} \alpha$ line emission is ubiquitous in pulsating red giants (e.g., McDonald \& van Loon 2007). Bessell et al. (1989) found that the temperature structure generated by Bowen's model does not produce a spectrum that resembles observed spectra of Miratype variables, questioning the efficacy of pulsation in levitating atmospheric layers.

Metal-poor AGB stars need longer to evolve before becoming sufficiently cool and strong pulsators, delaying the dustdriven wind stage (Bowen \& Willson 1991). This gives the core more time to grow, yielding a more massive white dwarf remnant. The observed differences between the white dwarf mass functions in the Magellanic Clouds and the Milky Way are very small (Villaver et al. 2004b, 2007; Williams 2007), implying that the dust-driven wind can neither be delayed nor weakened much.

\subsection{Implications for the enrichment and chemical mixing of the metal-poor interstellar medium}

It is important for understanding the continued star formation and chemical evolution of the Magellanic Clouds to establish which of the cool giants contribute most to the replenishment of the Magellanic Clouds' ISM, and how their material is mixed with the pre-existing ISM.

Carbon stars are plentiful due to a favourable IMF, and lower-metallicity star-forming galaxies are known to host larger carbon star populations (Groenewegen 1999). Carbon stars easily produce molecules and dust even at the low metallicity of the Magellanic Clouds. These molecules will have been destroyed by the interstellar radiation field (ISRF) before they could take part in the chemistry within molecular clouds that influences star formation. But they do supply the ISM with primary carbon. This could push the $\mathrm{CO}$ formation within molecular clouds to be limited by the oxygen abundance, and the formation of complex molecules to be dominated by carbonaceous chemistry.

Carbon stars in the Magellanic Clouds appear to produce less dust than their metal-richer siblings, although this is not conclusive until more $\mathrm{CO}$ detections of metal-poor carbon stars become available to measure their wind speeds and dust-to-gas ratios. Either way, carbon-rich dust has a fairly low condensation temperature and a high opacity, which means that it captures photons and disintegrates with much ease. This may explain the ubiquitous observation of PAHs in photon-dominated regions, both at the interfaces between $\mathrm{H}$ II regions and molecular clouds and between the central star and detached dust envelope within post-AGB objects. Hence the production of carbonrich dust may be of little consequence for the ISM. Although the seeds $\left(\mathrm{TiC}_{2}\right.$, possibly $\left.\mathrm{SiC}\right)$ may survive and participate in grain growth, the coatings will be a mixture of oxygen-rich and carbon-rich condensates.

The massive AGB stars and especially the RSGs that dominate the oxygen-rich dust production stray less far from their natal molecular clouds than carbon stars do, so the dust and gas they produce will participate in molecular clouds shielding from the ISRF and chemistry sooner than dust and gas from carbon stars. Meteoritic data suggest that chemical inhomogeneities exist in the Galactic ISM (Nittler et al. 2008). The slower winds of metal-poor red giants (Marshall et al. 2004; Mauron 2008) result in longer mixing timescales, also because they carry less momentum and thus stall more easily. This causes more localised chemical enrichment and continued star formation. The mixing of the material injected by carbon stars and low-mass oxygenrich AGB stars is due in part by ram-pressure stripping of their circumstellar envelopes (Villaver et al. 2004a; Wareing et al. 2006) as they travel through the ISM at speeds of tens of $\mathrm{km} \mathrm{s}^{-1}$, well in excess of their wind speeds. Younger, less dynamically relaxed populations are found within several $\mathrm{km} \mathrm{s}^{-1}$ from the local ISM velocity (e.g., van Loon et al. 2001). 


\section{Summary of conclusions}

We presented 2.9-4.1 $\mu \mathrm{m}$ spectra, obtained with ISAAC at the ESO/VLT, of a sample of dusty carbon stars, oxygen-rich AGB stars and red supergiants in the SMC. Strong absorption bands mainly due to acetylene are seen in the spectra of carbon stars, whilst many of the oxygen-rich stars too show strong molecular absorption. $\mathrm{OH}$ and/or $\mathrm{SiO}$ is detected in some oxygen-rich AGB stars and red supergiants, with the $\mathrm{SiO}$ sometimes seen in emission.

We also presented a spectrum of the post-AGB object MSX SMC 29, which displays very strong PAH emission. Absorption from aliphatic bonds may have been detected, which would point at the PAHs being released through photodestruction of the grains in the relic AGB envelope. The spectra of two R CrB-type stars show no molecular features but only a dust emission continuum. We suggest that these may be "protopost-AGB" objects.

The properties of the molecular bands in the SMC sample were compared with similar data in the LMC. The molecular absorption in SMC carbon stars is as strong (but not stronger) as that in LMC carbon stars, although there appears to be more cold molecular gas in the LMC carbon stars. Less conclusive results are obtained for the oxygen-rich stars. Veiling by dust emission is clearly more important in the LMC than in the SMC, suggesting that dust formation is less efficient at lower metallicity. The fact that this is seen also in the carbon stars suggests that the formation of dust in the molecular atmosphere relies on an intermediary agent, e.g., a nucleation seed based on a secondary element such as titanium or silicon. The lower dust content of oxygen-rich stars may also be caused by lower abundances of secondary elements, in the nucleation seed or the condensable molecular material.

The pulsation properties of a sample of AGB stars and red supergiants in the LMC were used to show that the mass-loss rate is likely determined more critically by the star's ability to levitate the molecular atmosphere through pulsation, than by the star's ability to drive the elevated molecular layers away through radiation pressure on the dust (unless dust does not form at all).

The slower winds of less dusty metal-poor AGB stars and red supergiants were discussed in the context of the enrichment and mixing of the mass loss with the ISM. Chemical inhomogeneities and small-scale structure in the dusty ISM are suggested to be more likely in metal-poor environments.

As a by-product of this programme, we obtained spectra of several Young Stellar Objects. These include the first detections of water ice in such objects in the SMC.

Acknowledgements. We wish to thank the astronomers and telescope operators at ESO Paranal for the excellent support during our observations, and an anonymous referee for her/his helpful suggestions. M.C. thanks NASA for supporting his participation in this work through contract JPL 1320707 with UC Berkeley. This publication makes use of data products from the Two Micron All Sky Survey, which is a joint project of the University of Massachusetts and the Infrared Processing and Analysis Center/California Institute of Technology, funded by the National Aeronautics and Space Administration and the National Science Foundation. This research has made use of the SIMBAD database, operated at CDS, Strasbourg, France. Based on observations made with the European Southern Observatory telescopes obtained from the ESO/ST-ECF Science Archive Facility.

\section{Appendix A: Galactic B-type emission-line stars}

As part of the telluric standard star calibration plan, three Galactic B-type stars were observed on 20/21 September 2005 that are now identified in the Simbad database as emissionline stars: HD 47054 (HIP 31583) of type B8 Ve, HD 71510

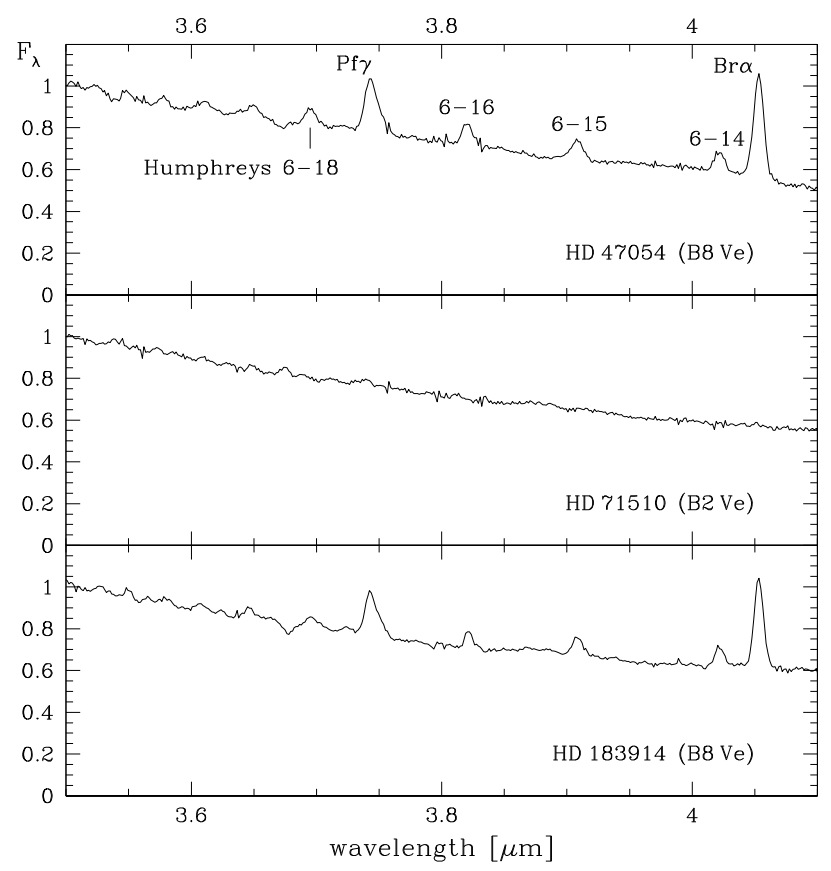

Fig. A.1. Spectra of three Galactic B-type emission-line stars.

(HIP 41296) of type B2 Ve, and HD 183914 (HIP 095951) of type B8 Ve.

The spectra of the two B8 stars clearly show hydrogen emission lines from the Brackett, Pfund and Humphreys series, but the spectrum of the hotter B2 star is completely featureless (Fig. A.1).

\section{References}

Bernatowicz, T. J., Amari, S., Zinner, E. K., \& Lewis, R. S. 1991, ApJ, 373, L73 Bessell, M. S., Brett, J. M., Wood, P. R., \& Scholz, M. 1989, A\&A, 213, 209

Blanco, V. M., Blanco, B. M., \& McCarthy, M. F. 1980, ApJ, 242, 938

Blanco, V. M., Frogel, J. A., \& McCarthy, M. F. 1981, PASP, 93, 532

Boothroyd, A. I., \& Sackmann, I.-J. 1992, ApJ, 393, L21

Boothroyd, A. I., Sackmann, I.-J., \& Ahern, S. C. 1993, ApJ, 416, 762

Bowen, G. H. 1988, ApJ, 329, 299

Bowen, G. H., \& Willson, L. A. 1991, ApJ, 375, L53

Cioni, M.-R. L., Blommaert, J. A. D. L., Groenewegen, M. A. T., et al. 2003, A\&A, 406, 51

Clayton, G. C. 1996, PASP, 108, 225

Clayton, G. C., Geballe, T. R., Herwig, F., Fryer, C., \& Asplund, M. 2007, ApJ, 662,120

Decin, L., \& Eriksson, K. 2007, A\&A, 472, 1041

de Freitas Pacheco, J. A., Barbuy, B., \& Idiart, T. 1998, A\&A, 332, 19

Elias, J. H., Frogel, J. A., \& Humphreys, R. M. 1985, ApJS, 57, 91

Ferrarotti, A. S., \& Gail, H.-P. 2006, A\&A, 447, 553

Filipović, M. D., Bohlsen, T., Reid, W., et al. 2002, MNRAS, 335, 1085

Fleischer, A. J., Gauger, A., \& Sedlmayr, E. 1992, A\&A, 266, 321

Frost, C. A., Cannon, R. C., Lattanzio, J. C., Wood, P. R., \& Forestini, M. 1998, A\&A, 332, L17

Gail, H.-P., \& Sedlmayr, E. 1988, A\&A, 206, 153

Groenewegen, M. A. T. 1999, in Asymptotic Giant Branch Stars, ed. T. Le Bertre, A. Lèbre, \& C. Waelkens, IAUS, 191, 535

Groenewegen, M. A. T., \& Blommaert, J. A. D. L. 1998, A\&A, 332, 25

Groenewegen, M. A. T., Smith, C. H., Wood, P. R., Omont, A., \& Fujiyoshi, T. 1995, ApJ, 449, L119

Groenewegen, M. A. T., Oudmaijer, R. D., \& Ludwig, H.-G. 1997, MNRAS, 292, 686

Groenewegen, M. A. T., Blommaert, J. A. D. L., Cioni, M.-R. L., et al. 2000, Mem. SAI, 71, 639

Groenewegen, M. A. T., Wood, P. R., Sloan, G. C., et al. 2007, MNRAS, 376, 313

Hodge, P. W. 1974, PASP, 86, 263

Höfner, S., \& Andersen, A. C. 2007, A\&A, 465, L39

Höfner, S., Feuchtinger, M. U., \& Dorfi, E. A. 1995, A\&A, 297, 815

Hron, J., Loidl, R., Höfner, S., et al. 1998, A\&A, 335, L69 
Jones, T. W., \& Merrill, K. M. 1976, ApJ, 209, 509

Judge, P. G., \& Stencel, R. E. 1991, ApJ, 371, 357

Kraemer, K. E., Sloan, G. C., Wood, P. R., Price, S. D., \& Egan, M. P. 2005, ApJ, 631, L147

Kraemer, K. E., Sloan, G. C., Bernard-Salas, J., et al. 2006, ApJ, 652, L25 Lagadec, E., Zijlstra, A. A., Sloan, G. C., et al. 2007, MNRAS, 376, 1270

Lambert, D. L., Rao, N. K., Pandey, G., \& Ivans, I. I. 2001, ApJ, 555, 925

Le Bertre, T., Tanaka, M., Yamamura, I., Murakami, H., \& MacConnell, D. J. 2005, PASP, 117, 199

Lloyd Evans, T. 1980a, MNRAS, 193, 87

Lloyd Evans, T. 1983, MNRAS, 204, 985

Loup, C., Zijlstra, A. A., Waters, L. B. F. M., \& Groenewegen, M. A. T. 1997, A\&AS, 125, 419

Marigo, P., Girardi, L., \& Chiosi, C. 1996, A\&A, 316, L1

Marigo, P., Girardi, L., \& Bressan, A. 1999, A\&A, 344, 123

Marshall, J. R., van Loon, J. Th., Matsuura, M., et al. 2004, MNRAS, 355, 1348

Matsuura, M., Zijlstra, A. A., van Loon, J. Th., et al. 2005, A\&A, 434, 691

Matsuura, M., Wood, P. R., Sloan, G. C., et al. 2006, MNRAS, 371, 415

Mattsson, L., Wahlin, R., Höfner, S., \& Eriksson, K. 2008, A\&A, 484, L5

Mauron, N. 2008, A\&A, 482, 151

McDonald, I., \& van Loon, J. Th. 2007, A\&A, 476, 1261

Merrill, K. M., \& Stein, W. A. 1976, PASP, 88, 285

Nittler, L. R., Alexander, C. M. O’D., Gallino, R., et al. 2008, ApJ, in press

Ohnaka, K., Driebe, T., Weigelt, G., \& Wittkowski, M. 2007, A\&A, 466, 1099

Ohnaka, K., Driebe, T., Hofmann, K.-H., Weigelt, G., \& Wittkowski, M. 2008, A\&A, 484, 371

Oliveira, J. M., van Loon, J. Th., Stanimirović, S., \& Zijlstra, A. A. 2006, MNRAS, 372, 1509

Oliveira, J. M., et al. 2008, in preparation

Rebeirot, E., Azzopardi, M., \& Westerlund, B. E. 1993, A\&AS, 97, 603

Rinsland, C. P., \& Wing, R. F. 1982, ApJ, 262, 201

Schwenke, D. W. 1998, in Chemistry and Physics of Molecules and Grains in Space, Faraday Discussions, 109, The Faraday Division of the Royal Society of Chemistry, London, 321

Skrutskie, M. F., Cutri, R. M., Stiening, R., et al. 2006, AJ, 131, 1163

Sloan, G. C., \& Price, S. D. 1998, ApJS, 119, 141

Sloan, G. C., Kraemer, K. E., Matsuura, M., et al. 2006, ApJ, 645, 1118

Sloan, G. C., Jura, M., Duley, W. W., et al. 2007, ApJ, 664, 1144

Sloan, G. C., et al. 2008, ApJ, submitted

Smith, R. G., Sellgren, K., \& Tokunaga, A. T. 1988, ApJ, 334, 209

Smith, V. V., Plez, B., Lambert, D. L., \& Lubowich, D. A. 1995, ApJ, 441, 735

Stanghellini, L., Garci'a-Lario, P., Garci'a-Herna'ndez, D. A., et al. 2007, ApJ, 671,1669
Tanabé, T., Nishida, S., Matsumoto, S., et al. 1997, Nature, 385, 509

Tanabé, T., Nishida, S., Nakada, Y., et al. 1999, IAUS, 191, 573

Tanabé, T., Kučinskas, A., Nakada, Y., Onaka, T., \& Sauvage, M. 2004, ApJS, 155,401

Trams, N. R., van Loon, J. Th., Waters, L. B. F. M., et al. 1999, A\&A, 346, 843

Tsuji, T., Ohnaka, K., Aoki, W., \& Yamamura, I. 1997, A\&A, 320, L1

van Loon, J. Th. 2000, A\&A, 354, 125

van Loon, J. Th. 2002, in Radial and Nonradial Pulsations as Probes of Stellar

Physics, ed. C. Aerts, T. R. Bedding, \& J. Christensen-Dalsgaard, ASPC, 259, 548

van Loon, J. Th. 2006, in Stellar Evolution at Low Metallicity: Mass Loss,

Explosions, Cosmology, ed. H. J. G. L. M. Lamers, N. Langer, T. Nugis, \& K. Annuk, ASPC, 353, 211

van Loon, J. Th., Zijlstra, A. A., \& Groenewegen, M. A. T. 1999a, A\&A, 346, 805

van Loon, J. Th., Groenewegen, M. A. T., de Koter, A., et al. 1999b, A\&A, 351, 559

van Loon, J. Th., Zijlstra, A. A., Bujarrabal, V., \& Nyman, L.-Å. 2001, A\&A, 368,950

van Loon, J. Th., Cioni, M.-R. L., Zijlstra, A. A., \& Loup, C. 2005a, A\&A, 438, 273

van Loon, J. Th., Marshall, J. R., \& Zijlstra, A. A. 2005b, A\&A, 442, 597

van Loon, J. Th., Oliveira, J. M., Wood, P. R., et al. 2005c, MNRAS, 364, L71

van Loon, J. Th., Marshall, J. R., Cohen, M., et al. 2006, A\&A, 447, 971

Villaver, E., Garcí-Segura, G., \& Manchado, A. 2004a, RMxAC, 22, 140

Villaver, E., Stanghellini, L., \& Shaw, R. A. 2004b, ApJ, 614, 716

Villaver, E., Stanghellini, L., \& Shaw, R. A. 2007, ApJ, 656, 831

Vollmer, C., Hoppe, P., Brenker, F., \& Palme, H. 2006, in 37th Annual Lunar and Planetary Science Conference, \#1284

Walker, M. F. 1972, MNRAS, 159, 379

Wallace, L., \& Hinkle, K. 2002, AJ, 124, 3393

Wareing, C. 2006, MNRAS, 372, L63

Westerlund, B. E. 1997, in The Magellanic Clouds, Camb. Astrophys. Ser., 29

Whitelock, P. A., Feast, M. W., Menzies, J. W., \& Catchpole, R. M. 1989, MNRAS, 238, 769

Whitelock, P., Menzies, J., Feast, M., et al. 1994, MNRAS, 267, 711

Williams, K. A. 2007, in 15th European Workshop on White Dwarfs, ed. R. Napiwotzki, \& M. Burleigh, ASPC, 372, 85

Woitke, P. 2006, A\&A, 460, L9

Wood, P. R., Whiteoak, J. B., Hughes, S. M. G., et al. 1992, ApJ, 397, 552

Yamamura, I., de Jong, T., \& Cami, J. 1999, A\&A, 348, L55

Zijlstra, A. A., Matsuura, M., Wood, P. R., et al. 2006, MNRAS, 370, 1961 